MATHEMATICS OF COMPUTATION

Volume 74, Number 252, Pages 1843-1870

S 0025-5718(05)01763-1

Article electronically published on June 7, 2005

\title{
STRONG STIELTJES DISTRIBUTIONS AND ORTHOGONAL LAURENT POLYNOMIALS WITH APPLICATIONS TO QUADRATURES AND PADÉ APPROXIMATION
}

\author{
C. DÍAZ-MENDOZA, P. GONZÁLEZ-VERA, AND M. JIMÉNEZ-PAIZ
}

\begin{abstract}
Starting from a strong Stieltjes distribution $\phi$, general sequences of orthogonal Laurent polynomials are introduced and some of their most relevant algebraic properties are studied. From this perspective, the connection between certain quadrature formulas associated with the distribution $\phi$ and two-point Padé approximants to the Stieltjes transform of $\phi$ is revisited. Finally, illustrative numerical examples are discussed.
\end{abstract}

\section{INTRODUCTION}

Let $-\infty \leq a<b \leq+\infty$ and let $\phi$ be a real, bounded and nondecreasing function with infinitely many points of increase on $(a, b)$, such that the moments

$$
m_{k}=\int_{a}^{b} t^{k} d \phi(t)
$$

exist for $k=0, \pm 1, \pm 2, \ldots$ Under these conditions $\phi$ is called a strong distribution on $(a, b)$. If the integrals (1.1) only exist for $k=0,1, \ldots$, it will be said that $\phi$ is a classical distribution. When $0 \leq a<b \leq+\infty$, $\phi$ will be called a strong Stieltjes distribution. These distributions were earlier introduced in 1980 by Jones et al. in connection with the so-called strong Stieltjes moment problem [26] and represent an extension of the classical Stieltjes moment problem 34 .

In this paper we shall be mainly concerned with strong Stieltjes distributions $\phi$ in order to estimate integrals of the form

$$
I_{\phi}(f)=\int_{a}^{b} f(t) d \phi(t)
$$

where $f$ is a function, with possible singularities located at the origin and/or infinity and such that $I_{\phi}(f)$ exists properly or improperly. For this purpose, we shall

Received by the editor May 5, 2003 and, in revised form, May 4, 2004.

2000 Mathematics Subject Classification. Primary 42C05, 41A55.

Key words and phrases. Strong Stieltjes distributions, orthogonal Laurent polynomials, quadrature formulas, Stieltjes transform, two-point Padé approximants.

This work was supported by the Scientific Research Projects of the Ministerio de Ciencia y Tecnología and Comunidad Autónoma de Canarias under contracts BFM2001-3411 and PI 2002/136, respectively.

(C)2005 American Mathematical Society Reverts to public domain 28 years from publication 
consider $n$-point quadrature of the form

$$
I_{n}(f)=\sum_{j=1}^{n} \lambda_{j} f\left(x_{j}\right)
$$

with nodes $\left\{x_{j}\right\}_{j=1}^{n} \subset(a, b), x_{j} \neq x_{k}$ for $j \neq k$ and positive weights $\left\{A_{j}\right\}_{j=1}^{n}$. Special attention will be paid for the case

$$
f(x)=f(x, z)=\frac{1}{z-x},
$$

$z$ being a parameter in $\mathbb{C} \backslash[a, b]$, which gives rise to the two-point Padé approximants to the Stieltjes transform $F_{\phi}(z)=I_{\phi}\left(\frac{1}{z-.}\right)$ of the distribution $\phi$, i.e.,

$$
F_{\phi}(z)=\int_{a}^{b} \frac{d \phi(t)}{z-t}, z \notin[a, b],
$$

when an appropriate selection of the nodes has been made. Here, the theory of the orthogonal Laurent polynomials will be the basic tool in a way similar to how the theory on orthogonal polynomials represents the basis for Gaussian quadrature formulas, Padé approximants at $\infty$ and other related topics (see 14]). Indeed, a theory on orthogonal Laurent polynomials started to make its appearance (25]) after the paper [26]. Since then, several articles have been written on this topic; see, e.g., [12, 22, 30. In most of these contributions the main aim consists of displaying the closest parallelism between the properties satisfied by the usual orthogonal polynomials and the orthogonal Laurent polynomials (as clearly indicated in [12, where the approach adopted there roughly parallels the one used by T.S. Chihara in the standard text [1]).

In this respect, in all the papers mentioned above, a special "ordering" in the space of the Laurent polynomials is previously established; however, from this approach, the properties of the usual orthogonal polynomials cannot be recovered (see [33]). So, this is just the starting point of the present paper: to develop a general theory on some aspects of orthogonal Laurent polynomials, with special emphasis on quadrature and Padé approximation, so that known results on either orthogonal polynomials or certain sequences of orthogonal Laurent polynomials already studied can now be deduced in a straightforward way. For an alternative approach on this topic, via orthogonal polynomials with respect to varying distributions, see [27] and also the series of papers [3, 4, 5, 6, by some of the present authors. On the other hand, related results can be found in 31 and 32 in connection with certain classes of continued fractions. For a complete survey on this topic see 23 ] and references found therein. Results concerning more general rational functions with prescribed poles than L-polynomials have recently been given in [8].

The paper is organized as follows. In Section 2, preliminary results on general sequences of orthogonal Laurent polynomials are provided. The main contribution of the paper is given in Section 3 concerning a Christoffel-Darboux identity and a three-term recurrence relation. As a result an interlacing property of the zeros of the orthogonal Laurent polynomials is also deduced. Section 4 and 5 are respectively dedicated to quadratures and two-point Padé approximants displaying the known closest connection between both topics. Finally, in Section 6 illustrative numerical examples are given. 


\section{ORthogonal LAURENT POLYNOMIALS}

Given two integers $p$ and $q$ such that $p \leq q$, by a Laurent polynomial (or Lpolynomial for short) we mean a function of the form

$$
L(x)=\sum_{j=p}^{q} \alpha_{j} x^{j}, \alpha_{j} \in \mathbb{R} .
$$

$\Delta_{p, q}$ will be the linear space of these functions, i.e.,

$$
\Delta_{p, q}=\operatorname{span}\left\{x^{j}: p \leq j \leq q\right\},
$$

which is a subspace of the linear space of all L-polynomials $\Delta=\operatorname{span}\left\{x^{k}: k \in \mathbb{Z}\right\}$.

Observe that $\operatorname{dim}\left(\Delta_{p, q}\right)=q-p+1$ and that if $\mathbb{P}_{k}$ denotes the linear space of polynomials of degree at most $k$, then $\mathbb{P}_{k}=\Delta_{0, k}$. Finally, $\mathbb{P}$ will denote the linear space of all algebraic polynomials.

Let $\phi$ be a strong Stieltjes distribution, from now on SSD, so that one can define an inner product on $\Delta$,

$$
\langle L, M\rangle_{\phi}=\int_{a}^{b} L(t) M(t) d \phi(t), \quad L, M \in \Delta
$$

(since we are going to deal with real functions one does not need complex conjugation in (2.1)).

In order to obtain an appropriate orthogonal basis in $\Delta$ with respect to (2.1), a certain nested sequence of linear subspaces of $\Delta$, similar to the sequence $\left\{\mathbb{P}_{n}\right\}_{n \geq 0}$ in $\mathbb{P}$, will first be required. This will be done starting from a nondecreasing sequence of nonnegative integers $\{p(n)\}_{n \geq 0}$ such that for each $n, 0 \leq p(n) \leq n$ and $p(n)-$ $p(n-1)=s(n) \in\{0,1\}$. Now, set

$$
\mathcal{L}_{n}=\Delta_{-p(n), q(n)}=\operatorname{span}\left\{x^{j}:-p(n) \leq j \leq q(n)\right\}, \quad p(n)+q(n)=n,
$$

and $\mathcal{L}=\bigcup_{n \geq 0} \mathcal{L}_{n}$. Thus, one sees that $\mathcal{L}_{n} \subset \mathcal{L}_{n+1}$ and that $\operatorname{dim}\left(\mathcal{L}_{n}\right)=n+1$. In this case, it will be said that the sequence $\{p(n)\}_{n \geq 0}$ has induced an "ordering" in $\mathcal{L}$. Furthermore, $\mathcal{L}=\Delta$ if and only if, $\lim _{n \rightarrow \infty} p(n)=\lim _{n \rightarrow \infty} q(n)=\infty$.

Moreover, the set of functions

$$
\frac{1}{x^{p(n)}}, \frac{1}{x^{p(n)-1}}, \ldots, \frac{1}{x}, 1, x, \ldots, x^{q(n)-1}, x^{q(n)}
$$

represents a Chebyshev system on any interval $(c, d) \subset \mathbb{R}$, such that $0 \notin(c, d)$. Hence, it exhibits the same interpolation properties as the usual polynomials. An adequate selection of $\{p(n)\}_{n \geq 0}$ would be motivated by the singularities of the function to be approximated near the origin and/or infinity.

Let $R_{n} \in \mathcal{L}_{n} \backslash \mathcal{L}_{n-1}, R_{n}(x)=\sum_{j=-p(n)}^{q(n)} r_{j, n} x^{j}$, and set

$$
\kappa_{n}=\left\{\begin{array}{lll}
r_{-p(n), n} & \text { if } & p(n)>p(n-1), \\
r_{q(n), n} & \text { if } & p(n)=p(n-1) .
\end{array}\right.
$$

Then, $\kappa_{n} \neq 0$ is called the leading coefficient of $R_{n}$.

On the other hand, by applying the Gram-Schmidt orthogonalization process to the basis $\left\{x^{j}:-p(n) \leq j \leq q(n)\right\}$ of $\mathcal{L}_{n}$, an orthogonal basis $\left\{\varphi_{0}, \varphi_{1}, \ldots, \varphi_{n}\right\}$ can be obtained. When the process is repeated for each $n=1,2, \ldots$, an essentially unique sequence $\left\{\varphi_{n}\right\}_{n \geq 0}$ is deduced satisfying

$$
\varphi_{n} \in \mathcal{L}_{n} \backslash \mathcal{L}_{n-1}, \quad\left\langle\varphi_{n}, L\right\rangle_{\phi}=0, \forall L \in \mathcal{L}_{n-1}, n \geq 1 \quad \text { and } \quad \varphi_{0} \equiv 1,
$$


i.e., $\varphi_{n} \perp \mathcal{L}_{n-1}$. When $\kappa_{n}=1$, for each $n \geq 0,\left\{\varphi_{n}\right\}_{n \geq 0}$ is said to be the sequence of monic orthogonal Laurent polynomials with respect to the SSD $\phi$ associated with $\{p(n)\}_{n \geq 0}$.

Clearly, for $m$ and $n$ nonnegative integers it holds that $\left\langle\varphi_{n}, \varphi_{m}\right\rangle_{\phi}=\delta_{n, m} \frac{1}{\gamma_{n}^{2}}$ with $\gamma_{n}>0$. When $\gamma_{n}=1$, for each $n \geq 0$, the system $\left\{\varphi_{n}\right\}_{n \geq 0}$ is orthonormal and is uniquely determined up to a sign.

Making use of standard arguments as in the polynomial situation, we get the following proposition.

Proposition 2.1. Let $\left\{\varphi_{n}\right\}_{n \geq 0}$ be a sequence of orthogonal L-polynomial with respect to the $S S D \phi$ on $\mathcal{L}$ associated with a nondecreasing sequence $\{p(n)\}_{n \geq 0}$ of nonnegative integers such that $0 \leq p(n) \leq n$ and $p(n)-p(n-1)=s(n) \in\{0,1\}$ for each $n$. Then, $\varphi_{n}$ has exactly $n$ distinct zeros in $(a, b)$.

Proof. Let $\varsigma_{1, n}, \varsigma_{2, n}, \ldots, \varsigma_{k, n}$ be the distinct zeros of $\varphi_{n}$ on $(a, b)$ with odd multiplicity. Assume that $k<n$ and set

$$
R_{k}(x)=\frac{\left(x-\varsigma_{1, n}\right)\left(x-\varsigma_{2, n}\right) \cdots\left(x-\varsigma_{k, n}\right)}{x^{p(k)}} \in \mathcal{L}_{k} .
$$

Since $0 \leq a<b \leq \infty, \varphi_{n}(x) R_{k}(x)$ has constant sign on $(a, b)$. Hence

$$
\int_{a}^{b} \varphi_{n}(t) R_{k}(t) d \phi(t) \neq 0
$$

and a contradiction arises since $\varphi_{n} \perp \mathcal{L}_{k}$. Hence $k=n$.

From this proposition it is clear that if we write $\varphi_{n}(x)=\frac{p_{n}(x)}{x^{p(n)}}$, then $p_{n}$ is a polynomial of exact degree $n$ such that $p_{n}(0) \neq 0$. Thus, one could also normalize the sequence $\left\{\varphi_{n}\right\}_{n \geq 0}$ by imposing that for each $n, p_{n}$ is a monic polynomial of degree $n$. Observe that it does not mean that $\varphi_{n}$ is monic according to the above definition. Such a sequence of orthogonal L-polynomials will be denoted by $\left\{\psi_{n}\right\}_{n \geq 0}$ so that by setting $\psi_{n}(x)=\sum_{j=-p(n)}^{q(n)} r_{j, n} x^{j}$, we have $r_{q(n), n}=1$ and $r_{-p(n), n}=$ $(-1)^{n} \prod_{j=1}^{n} x_{j, n}$, where $\left\{x_{j, n}\right\}_{j=1}^{n}$ are the zeros of $\psi_{n}$.

Assume now that $\left\{\Psi_{n}\right\}_{n \geq 0}$ represents the sequence of orthonormal L-polynomials with respect to the SSD $\phi$ on $\mathcal{L}$ associated with the ordering induced by $\{p(n)\}_{n \geq 0}$ and obtained from $\left\{\psi_{n}\right\}_{n \geq 0}$. Then, we define ( $z$ and $y$ are both different from zero)

$$
\mathcal{K}_{n}(z, y)=\sum_{k=0}^{n} \Psi_{k}(z) \Psi_{k}(y)
$$

Thus, if $L$ is an L-polynomial in $\mathcal{L}_{n}$, one has

$$
L(x)=\sum_{k=0}^{n} a_{k} \Psi_{k}(x) \quad \text { with } \quad a_{k}=\left\langle L, \Psi_{k}\right\rangle_{\phi}, k=0,1, \ldots, n .
$$

Hence, it follows that

$$
\left\langle\mathcal{K}_{n}(\cdot, y), L\right\rangle_{\phi}=\int_{a}^{b} \mathcal{K}_{n}(t, y) L(t) d \phi(t)=L(y) .
$$

For this reason, we shall call $\mathcal{K}_{n}(z, y)$ given by (2.2) the reproducing kernel of $\mathcal{L}_{n}$. Property (2.3) will be used in the next Section. 
To conclude, we will also introduce certain L-polynomials associated with the sequence $\left\{\psi_{n}\right\}_{n \geq 0}$ (the second kind L-polynomial), namely

$$
\sigma_{n}(x)=\int_{a}^{b} \frac{\psi_{n}(x)-\psi_{n}(t)}{x-t} d \phi(t), n=0,1, \ldots
$$

When $\psi_{n}$ is replaced by $\Psi_{n}$, we use the notation $\Sigma_{n}$ instead of $\sigma_{n}$. Thus, $\sigma_{n}(x)=$ $\frac{q_{n}(x)}{x^{p(n)}} \in \mathcal{L}_{n}$, with $q_{n} \in \mathbb{P}_{n-1}$ for $n \geq 1$ and $\sigma_{0} \equiv 0$. These L-polynomials will appear in Section 5 concerning two-point Padé approximants. Clearly when $p(n)=$ $0, n=0,1, \ldots$, the usual second kind polynomials associated with a sequence of orthogonal polynomials immediately arise.

\section{THREE-TERM RECURRENCE RELATION And Christoffel-Darboux IDENTity}

From the theory of orthogonal polynomials, it is well known that a basic property is the three-term recurrence relation satisfied by a sequence of orthogonal polynomials with respect to a classical distribution along with the so-called ChristoffelDarboux identity.

Thus, taking into account that for $p(n)=0$ (hence $q(n)=n, n=0,1, \ldots$ ) the usual polynomials are recovered, one might wonder whether a three-term recurrence relation and a Christoffel-Darboux identity could also hold for an arbitrary "ordering" generated by an arbitrary sequence $\{p(n)\}_{n \geq 0}$. In this respect, a first positive partial answer has been given when dealing with $p(n)=E\left[\frac{n+1}{2}\right]$ (see [12, 30]), where $E[a]$ denotes the integer part of $a$. Here it should also be noted that this choice of $p(n)$ is the basis of the previously established theory on orthogonal L-polynomials. For details, see, e.g., [16, 18, 19.

Thus, for our purposes we will start from an arbitrary nondecreasing sequence $\{p(n)\}_{n \geq 0}$ of nonnegative integers satisfying $0 \leq p(n) \leq n$ and $p(n)-p(n-1)=$ $s(n) \in\{0,1\}$ for each $n$. Let $\left\{\Psi_{n}\right\}_{n \geq 0}$ be the corresponding sequence of orthonormal L-polynomials for the SSD $d \phi$ on $\mathcal{L}$ associated with the ordering induced by $\{p(n)\}_{n \geq 0}$. Then the following result holds.

Theorem 3.1 (Christoffel-Darboux identity). For $z$ and $y$ real numbers both different from zero, one has

$\sum_{k=0}^{n} \Psi_{k}(z) \Psi_{k}(y)=C_{n}\left[\frac{z^{s(n+1)} \Psi_{n+1}(z) y^{s(n)} \Psi_{n}(y)-z^{s(n)} \Psi_{n}(z) y^{s(n+1)} \Psi_{n+1}(y)}{z-y}\right]$,

where $p(n)=s(n)+p(n-1)$ and $C_{n}>0, n \geq 1$. Furthermore, (3.1) also holds for $n=0$, with $s(0)=0$ and $C_{0}>0$ conveniently chosen.

Proof. Set $\mathcal{K}_{n}(z, y)=\sum_{k=0}^{n} \Psi_{k}(z) \Psi_{k}(y)$ and take $L \in \mathcal{L}_{n-1}$ for $n \geq 1$. Hence $\frac{(z-y) L(y)}{y^{s(n)}} \in \mathcal{L}_{n}($ in the variable $y)$.

Thus, by 2.3 one has

$$
\int_{a}^{b} \mathcal{K}_{n}(z, t) \frac{(z-t) L(t)}{t^{s(n)}} d \phi(t)=0, \quad \forall L \in \mathcal{L}_{n-1} .
$$

That is, $\mathcal{K}_{n}(z, y) \frac{(z-y)}{y^{s(n)}}$ is orthogonal to $\mathcal{L}_{n-1}$ (in the variable $y$ ). However, it should be observed that, in general, $\mathcal{K}_{n}(z, y) \frac{(z-y)}{y^{s(n)}} \notin \mathcal{L}_{n+1}$ (in the variable $y$ ) as desirable. 
Setting $\Delta s(n)=s(n+1)-s(n) \in\{-1,0,1\}($ recall $s(n)=p(n)-p(n-1) \in$ $\{0,1\})$, one can write

$$
\mathcal{K}_{n}(z, y) \frac{(z-y)}{y^{s(n)}}=\frac{\mathcal{K}_{n}(z, y)(z-y)}{y^{s(n+1)}} y^{\Delta s(n)} .
$$

Clearly, the function $\mathcal{K}_{n}(z, y) \frac{(z-y)}{y^{s(n+1)}} \in \mathcal{L}_{n+1}$ (in the variable $y$ ).

Assuming now that $\Delta s(n)=0$, it holds that

$$
\mathcal{K}_{n}(z, y) \frac{(z-y)}{y^{s(n)}} \in \mathcal{L}_{n+1} \quad \text { and } \quad \mathcal{K}_{n}(z, y) \frac{(z-y)}{y^{s(n)}} \perp \mathcal{L}_{n-1} .
$$

By (3.2) the Fourier sum of this function with respect to the orthonormal basis $\left\{\Psi_{n}\right\}_{n \geq 0}$ is of the form

$$
\mathcal{K}_{n}(z, y) \frac{(z-y)}{y^{s(n)}}=\tilde{a}_{n}(z) \Psi_{n+1}(y)+\tilde{b}_{n}(z) \Psi_{n}(y)
$$

or, equivalently,

$$
\mathcal{K}_{n}(z, y) \frac{(z-y)}{z^{s(n)} y^{s(n)}}=a_{n}(z) \Psi_{n+1}(y)+b_{n}(z) \Psi_{n}(y) .
$$

If we set $\Psi_{n}(x)=\frac{P_{n}(x)}{x^{p(n)}}$ where $P_{n} \in \mathbb{P}_{n}$ such that $P_{n}(x)=\gamma_{n} x^{n}+\cdots+P_{n}(0)$ with $\gamma_{n}>0$, then $(-1)^{n} P_{n}(0)>0$ and from (3.3), by comparing leading coefficients, one deduces that $a_{n}(z)=-(-1)^{s(n)} A_{n} \Psi_{n}(z)$ with

$$
A_{n}=s(n) \frac{P_{n}(0)}{P_{n+1}(0)}+(1-s(n)) \frac{\gamma_{n}}{\gamma_{n+1}} .
$$

Observe that $(-1)^{s(n)} A_{n}>0$. In short, by substituting this last expression in (3.3), one has

$$
\mathcal{K}_{n}(z, y) \frac{(z-y)}{z^{s(n)} y^{s(n)}}=-(-1)^{s(n)} A_{n} \Psi_{n}(z) \Psi_{n+1}(y)+b_{n}(z) \Psi_{n}(y) .
$$

If we carry on the same computations by exchanging $z$ and $y$, it follows that

$$
\mathcal{K}_{n}(z, y) \frac{(y-z)}{z^{s(n)} y^{s(n)}}=-(-1)^{s(n)} A_{n} \Psi_{n}(y) \Psi_{n+1}(z)+b_{n}(y) \Psi_{n}(z) .
$$

Subtracting both equalities, one obtains

$$
\begin{aligned}
2 \frac{\mathcal{K}_{n}(z, y)(z-y)}{z^{s(n)} y^{s(n)}}= & -(-1)^{s(n)} A_{n}\left[\Psi_{n}(z) \Psi_{n+1}(y)-\Psi_{n}(y) \Psi_{n+1}(z)\right] \\
& +\left[b_{n}(z) \Psi_{n}(y)-b_{n}(y) \Psi_{n}(z)\right] .
\end{aligned}
$$

Moreover, by (3.5) one sees that $b_{n}$ belongs to $\mathcal{L}_{n+1}$ and is orthogonal to $\mathcal{L}_{n-1}$. So,

$$
b_{n}(x)=\theta_{n} \Psi_{n+1}(x)+\mu_{n} \Psi_{n}(x), \theta_{n}, \mu_{n} \in \mathbb{R} .
$$

Furthermore, introducing $b_{n}$ in (3.5) and comparing leading coefficients results in $\theta_{n}=(-1)^{s(n)} A_{n}$. Hence,

$$
b_{n}(z) \Psi_{n}(y)-b_{n}(y) \Psi_{n}(z)=(-1)^{s(n)} A_{n}\left[\Psi_{n+1}(z) \Psi_{n}(y)-\Psi_{n}(z) \Psi_{n+1}(y)\right] .
$$


Thus, by (3.6) and (3.7), it follows that

$$
\mathcal{K}_{n}(z, y) \frac{(z-y)}{z^{s(n)} y^{s(n)}}=(-1)^{s(n)} A_{n}\left[\Psi_{n+1}(z) \Psi_{n}(y)-\Psi_{n}(z) \Psi_{n+1}(y)\right],
$$

yielding,

$\mathcal{K}_{n}(z, y)(z-y)=(-1)^{s(n)} A_{n}\left[z^{s(n)} \Psi_{n+1}(z) y^{s(n)} \Psi_{n}(y)-z^{s(n)} \Psi_{n}(z) y^{s(n)} \Psi_{n+1}(y)\right]$.

Assuming that $\Delta s(n)=0$ results in

$$
\mathcal{K}_{n}(z, y)=(-1)^{s(n)} A_{n}\left[\frac{z^{s(n+1)} \Psi_{n+1}(z) y^{s(n)} \Psi_{n}(y)-z^{s(n)} \Psi_{n}(z) y^{s(n+1)} \Psi_{n+1}(y)}{z-y}\right],
$$

if $C_{n}=(-1)^{s(n)} A_{n}$, the theorem is true under the assumption $\Delta s(n)=0$.

Next, let us analyze what happens when $\Delta s(n) \neq 0$. For this, define the subspace

$$
\tilde{\mathcal{L}}_{n+1}=\mathcal{L}_{n} \oplus \operatorname{span}\left\langle\frac{s(n)}{x^{p(n)+1}}+(1-s(n)) x^{q(n)+1}\right\rangle,
$$

so that

$$
\mathcal{K}_{n}(z, y) \frac{z-y}{y^{s(n)}} \in \tilde{\mathcal{L}}_{n+1}(\text { in the variable } y) .
$$

Let us now consider the finite sequence of nested subspaces

$$
\mathcal{L}_{0} \subset \mathcal{L}_{1} \subset \cdots \subset \mathcal{L}_{n} \subset \tilde{\mathcal{L}}_{n+1}
$$

and let $\left\{\tilde{\Psi}_{k}\right\}_{k=0}^{n+1}$ be an orthonormal basis for $\tilde{\mathcal{L}}_{n+1}$. Then, it holds that $\tilde{\Psi}_{k}=$ $\Psi_{k}, k=0,1, \ldots, n$, and $\tilde{\Psi}_{n+1} \in \tilde{\mathcal{L}}_{n+1} \backslash \mathcal{L}_{n}$. Actually, this process is equivalent to defining a new sequence $\{\tilde{p}(k)\}_{k=0}^{n+1}$ such that $\tilde{p}(k)=p(k), 0 \leq k \leq n$, and $\tilde{p}(n+1)=p(n)+s(n)$. Hence, $\tilde{s}(n+1)=\tilde{s}(n)=s(n)$ and $\Delta \tilde{s}(n)=0$ and the proof reduces to the situation above by substituting $\{p(k)\}_{k=0}^{n+1}$ for $\{\tilde{p}(k)\}_{k=0}^{n+1}$. Thus, by (3.8) and recalling that $\mathcal{K}_{n}(z, y) \frac{(z-y)}{z^{s(n)} y^{s(n)}} \perp \mathcal{L}_{n-1}$ (in the variable $y$ ), we can proceed as in the preceding situation to obtain

$$
\mathcal{K}_{n}(z, y) \frac{(z-y)}{z^{s(n)} y^{s(n)}}=(-1)^{s(n)} \tilde{A}_{n}\left[\tilde{\Psi}_{n+1}(z) \Psi_{n}(y)-\Psi_{n}(z) \tilde{\Psi}_{n+1}(y)\right],
$$

where $(-1)^{s(n)} \tilde{A}_{n}>0$ with $\tilde{A}_{n}=s(n) \frac{P_{n}(0)}{\tilde{P}_{n+1}(0)}+(1-s(n)) \frac{\gamma}{\tilde{\gamma}_{n+1}}$.

On the other hand, it can be easily checked that

$$
x^{\Delta s(n)} \Psi_{n+1}(x) \in \tilde{\mathcal{L}}_{n+1} \backslash \mathcal{L}_{n} .
$$

Hence, it follows that

$$
\tilde{\Psi}_{n+1}(x)=u_{n+1} x^{\Delta s(n)} \Psi_{n+1}(x)+F_{n}(x),
$$

with $u_{n+1}=s(n) \frac{\tilde{P}_{n+1}(0)}{P_{n+1}(0)}+(1-s(n)) \frac{\tilde{\gamma}_{n+1}}{\gamma_{n+1}} \neq 0$ where $\tilde{\Psi}_{n+1}(x)=\frac{\tilde{P}_{n+1}(x)}{x^{\tilde{p}(n+1)}}$ with $\tilde{P}_{n+1}(x)=\tilde{\gamma}_{n+1} x^{n+1}+\cdots+\tilde{P}_{n+1}(0)$ and $F_{n} \in \mathcal{L}_{n}$. Now, since $\Psi_{n+1} \perp \mathcal{L}_{n}$, we have

$$
x^{\Delta s(n)} \Psi_{n+1}(x) \perp \operatorname{span}\left\langle\frac{1}{x^{p(n)+\Delta s(n)}}, \ldots, x^{q(n)-\Delta s(n)}\right\rangle \supset \mathcal{L}_{n-1},
$$

which implies that

$$
x^{\Delta s(n)} \Psi_{n+1}(x) \perp \mathcal{L}_{n-1} .
$$


Thus, taking into account that $\tilde{\Psi}_{n+1} \perp \mathcal{L}_{n-1}$, from (3.10) and (3.11), we can deduce that $F_{n} \perp \mathcal{L}_{n-1}$, yielding

$$
\tilde{\Psi}_{n+1}(x)=u_{n+1} x^{\Delta s(n)} \Psi_{n+1}(x)+v_{n+1} \Psi_{n}(x) .
$$

So, from (3.9) and observing that $\tilde{A}_{n} u_{n+1}=A_{n}$ given by (3.4), it follows that

$$
\mathcal{K}_{n}(z, y) \frac{(z-y)}{z^{s(n)} y^{s(n)}}=(-1)^{s(n)} A_{n}\left[z^{\Delta s(n)} \Psi_{n+1}(z) \Psi_{n}(y)-\Psi_{n}(z) y^{\Delta s(n)} \Psi_{n+1}(y)\right] .
$$

From here, one has

$\mathcal{K}_{n}(z, y)=(-1)^{s(n)} A_{n}\left[\frac{z^{s(n+1)} \Psi_{n+1}(z) y^{s(n)} \Psi_{n}(y)-z^{s(n)} \Psi_{n}(z) y^{s(n+1)} \Psi_{n+1}(y)}{z-y}\right]$.

Finally, if $C_{n}=(-1)^{s(n)} A_{n}>0$, the proof is concluded.

Concerning a three-term recurrence relation, one can first prove the following:

Theorem 3.2. Let $\left\{\mathcal{L}_{n}\right\}_{n \geq 0} \subset \Delta$ be a sequence of nested subspaces of L-polynomials generated by a nondecreasing sequence of nonnegative integers $\{p(n)\}_{n \geq 0}$ such that $0 \leq p(n) \leq n$ and $p(n)-p(n-1)=s(n) \in\{0,1\}$ for each $n$. Let $\left\{S_{n}\right\}_{n \geq 0}$ be a sequence of L-polynomials satisfying $S_{n} \in \mathcal{L}_{n} \backslash \mathcal{L}_{n-1}\left(S_{0} \in \mathcal{L}_{0}\right)$ for $n \geq 1$, and

$$
\begin{aligned}
& \sum_{k=0}^{n} S_{k}(z) S_{k}(y)(z-y) \\
& \quad=C_{n}\left[z^{s(n+1)} S_{n+1}(z) y^{s(n)} S_{n}(y)-z^{s(n)} S_{n}(z) y^{s(n+1)} S_{n+1}(y)\right],
\end{aligned}
$$

for $n \geq 0$ with $C_{n} \neq 0$ and $s(0)=0$. Then, there exists a sequence $\left\{\Lambda_{n}\right\}_{n \geq 1} \subset \mathbb{R}$ such that

$$
\begin{aligned}
& C_{n} x^{s(n+1)} S_{n+1}(x) \\
& \quad=(-1)^{s(n)}\left[x^{1-2 s(n)}+\Lambda_{n}\right] x^{s(n)} S_{n}(x)-C_{n-1} x^{s(n-1)} S_{n-1}(x), \quad n \geq 1 .
\end{aligned}
$$

Proof. Let $\mathcal{M}_{n}(z, y)=\sum_{k=0}^{n} S_{k}(z) S_{k}(y)$. For $n \geq 1$, it follows that

$$
\begin{aligned}
\mathcal{M}_{n}(z, y)(z-y)=S_{n}(z) S_{n}(y)(z-y)+\mathcal{M}_{n-1}(z, y)(z-y) & =S_{n}(z) S_{n}(y)(z-y) \\
& +C_{n-1}\left[z^{s(n)} S_{n}(z) y^{s(n-1)} S_{n-1}(y)-z^{s(n-1)} S_{n-1}(z) y^{s(n)} S_{n}(y)\right] .
\end{aligned}
$$

That is,

$$
\begin{aligned}
C_{n}\left[z^{s(n+1)} S_{n+1}(z) y^{s(n)} S_{n}(y)-z^{s(n)} S_{n}(z) y^{s(n+1)} S_{n+1}(y)\right] \\
=S_{n}(z) S_{n}(y)(z-y) \\
\quad+C_{n-1}\left[z^{s(n)} S_{n}(z) y^{s(n-1)} S_{n-1}(y)-z^{s(n-1)} S_{n-1}(z) y^{s(n)} S_{n}(y)\right] .
\end{aligned}
$$

From here, it follows that

$$
\begin{aligned}
y^{s(n)} & S_{n}(y)\left[C_{n} z^{s(n+1)} S_{n+1}(z)-\frac{z}{y^{s(n)}} S_{n}(z)+C_{n-1} z^{s(n-1)} S_{n-1}(z)\right] \\
& =z^{s(n)} S_{n}(z)\left[C_{n} y^{s(n+1)} S_{n+1}(y)-\frac{y}{z^{s(n)}} S_{n}(y)+C_{n-1} y^{s(n-1)} S_{n-1}(y)\right],
\end{aligned}
$$


or equivalently,

$$
\begin{aligned}
& \frac{C_{n} z^{s(n+1)} S_{n+1}(z)+C_{n-1} z^{s(n-1)} S_{n-1}(z)}{z^{s(n)} S_{n}(z)}-\frac{z^{1-s(n)}}{y^{s(n)}} \\
& \quad=\frac{C_{n} y^{s(n+1)} S_{n+1}(y)+C_{n-1} y^{s(n-1)} S_{n-1}(y)}{y^{s(n)} S_{n}(y)}-\frac{y^{1-s(n)}}{z^{s(n)}} .
\end{aligned}
$$

Since

$$
\frac{z^{1-s(n)}}{y^{s(n)}}-\frac{y^{1-s(n)}}{z^{s(n)}}=\frac{z-y}{z^{s(n)} y^{s(n)}}=(-1)^{s(n)}\left(z^{1-2 s(n)}-y^{1-2 s(n)}\right),
$$

it follows that

$$
\begin{aligned}
& \frac{C_{n} z^{s(n+1)} S_{n+1}(z)+C_{n-1} z^{s(n-1)} S_{n-1}(z)}{z^{s(n)} S_{n}(z)}-(-1)^{s(n)} z^{1-2 s(n)} \\
& \quad=\frac{C_{n} y^{s(n+1)} S_{n+1}(y)+C_{n-1} y^{s(n-1)} S_{n-1}(y)}{y^{s(n)} S_{n}(y)}-(-1)^{s(n)} y^{1-2 s(n)} .
\end{aligned}
$$

From here, it necessarily follows that

$$
\frac{C_{n} x^{s(n+1)} S_{n+1}(x)+C_{n-1} x^{s(n-1)} S_{n-1}(x)-(-1)^{s(n)} x^{1-s(n)} S_{n}(x)}{x^{s(n)} S_{n}(x)}
$$

is a constant, say, $\tilde{\Lambda}_{n}$. The proof can be immediately concluded by taking $\Lambda_{n}=$ $(-1)^{s(n)} \tilde{\Lambda}_{n}$.

From Theorems 3.1 and 3.2, we can deduce the following:

Corollary 3.3 (Three-term recurrence relation). Let $\left\{\Psi_{k}\right\}_{k \in \mathbb{N}}$ be the sequence of orthonormal Laurent polynomials for the SSD $\phi$ with the ordering induced by a nondecreasing sequence $\{p(n)\}_{n \geq 0}$ of nonnegative integers such that $0 \leq p(n) \leq n$ and $p(n)-p(n-1)=s(n) \in\{0,1\}$ for each $n$. Then, there exist two sequences of positive real numbers $\left\{\Omega_{n}\right\}_{n \geq 1}$ and $\left\{C_{n}\right\}_{n \geq 0}$ such that for $n \geq 1$

$C_{n} z^{s(n+1)} \Psi_{n+1}(z)=(-1)^{s(n)}\left[z^{1-2 s(n)}-\Omega_{n}\right] z^{s(n)} \Psi_{n}(z)-C_{n-1} z^{s(n-1)} \Psi_{n-1}(z)$, with $s(0)=0$. Furthermore, (3.13) also holds for $n=0$ setting $C_{0}=\frac{1}{\sqrt{m_{0}} \gamma_{1}}$, $s(-1)=0, \Psi_{-1} \equiv 0, \Omega_{0}=\frac{m_{1-s(1)}}{m_{-s(1)}}$ and with $C_{-1}$ arbitrary (here $\left\{m_{k}\right\}_{k \in \mathbb{Z}}$ are the moments of $\phi)$.

Proof. Making use of Theorems 3.1 and 3.2, it only remains to prove that $\Omega_{n}>0$ for $n=1,2, \ldots$ Indeed, set $\Psi_{n}(x)=\frac{P_{n}(x)}{x^{p(n)}}$, where $P_{n}(x)=\gamma_{n} x^{n}+\cdots+P_{n}(0)$, such that $\gamma_{n}>0$ and $(-1)^{n} P_{n}(0)>0$. Then

$$
\begin{aligned}
& C_{n} P_{n+1}(z)+C_{n-1} z^{s(n-1)+s(n)} P_{n-1}(z) \\
& \quad=(-1)^{s(n)}\left[z^{1-s(n)}-\Omega_{n} z^{s(n)}\right] P_{n}(z), \quad n \geq 0 .
\end{aligned}
$$

Assume first that $s(n)=0$. Then taking $z=0$ in (3.14), one deduces

$$
\Omega_{n}=-\left[C_{n} \frac{P_{n+1}(0)}{P_{n}(0)}+(1-s(n-1)) C_{n-1} \frac{P_{n-1}(0)}{P_{n}(0)}\right]>0,
$$

since $C_{n}>0$ for all $n$.

On the other hand, by assuming $s(n)=1$ and considering $z=\infty$, it follows that

$$
\Omega_{n}=C_{n} \frac{\gamma_{n+1}}{\gamma_{n}}+C_{n-1} s(n-1) \frac{\gamma_{n-1}}{\gamma_{n}}>0 .
$$


Remark 3.4. Actually, relation (3.14) can also be read

$$
\begin{aligned}
C_{n} P_{n+1}(z)= & (-1)^{s(n)}\left[z^{1-2 s(n)}-\Omega_{n}\right] z^{s(n)} P_{n}(z) \\
& -C_{n-1} z^{s(n-1)+s(n)} P_{n-1}(z), \quad n \geq 0,
\end{aligned}
$$

with $P_{-1} \equiv 0, P_{0} \equiv \frac{1}{\sqrt{m_{0}}}$. Thus, when taking $p(n)=0$ for each $n$, we have $\mathcal{L}_{n}=\mathbb{P}_{n}$ and $\Psi_{n}=P_{n}$. Hence, from (3.15) we deduce the usual three-term recurrence for orthonormal polynomials.

On the other hand, as we have already mentioned, if we choose $p(n)=E\left[\frac{n+1}{2}\right]$, the known theory on orthogonal Laurent polynomials arises. In this case, a threeterm recurrence relation and a Christoffel-Darboux formula were earlier obtained (see [12, 25, 30]). There, from the orthogonality conditions a three-term recurrence relation is first deduced and then a Christoffel-Darboux identity is obtained. Here, and as we have seen, a different approach has been followed.

Now, for $p(n)=E\left[\frac{n+1}{2}\right]$,

$$
s(n)=p(n)-p(n-1)=E\left[\frac{n+1}{2}\right]-E\left[\frac{n}{2}\right]= \begin{cases}0 & \text { if } n \text { is even } \\ 1 & \text { if } n \text { is odd }\end{cases}
$$

Thus, $s(n)-s(n-1)=(-1)^{n+1}$ and $s(n+1)-s(n-1)=0$. Hence, 3.13) becomes

$$
C_{n} \Psi_{n+1}(z)=(-1)^{n}\left[1-z^{(-1)^{n+1}} \Omega_{n}\right] \Psi_{n}(z)-C_{n-1} \Psi_{n-1}(z) .
$$

In this case, from the orthogonality conditions for $\Psi_{n}$ it holds that

$$
\int_{a}^{b} P_{n}(t) t^{k} \frac{d \phi(t)}{t^{n}}=0, \quad k=0,1, \ldots, n-1 .
$$

Polynomials $\left\{P_{n}\right\}_{n=0}^{\infty}$ satisfying (3.16) have been extensively studied by Ranga et al. $($ 31, 32, 33]).

Remark 3.5. Concerning the Laurent polynomials $\Sigma_{n}$ associated with $\Psi_{n}$ as in (2.4), it can be proved they satisfy the same recurrence relation (3.13) but with different initial conditions. More precisely, for $n \geq 1$ it holds that

$$
C_{n} z^{s(n+1)} \Sigma_{n+1}(z)=(-1)^{s(n)}\left[z^{1-2 s(n)}-\Omega_{n}\right] z^{s(n)} \Sigma_{n}(z)-C_{n-1} z^{s(n-1)} \Sigma_{n-1}(z)
$$

with $\Sigma_{0}(z) \equiv 0$ and $\Sigma_{1}(z)=\frac{\gamma_{1} m_{-s(1)}}{(-z)^{s(1)}}$. Furthermore, (3.17) is also valid for $n=0$, considering $s(0)=s(-1)=0, \Sigma_{-1}(z) \equiv 1$ and adequately choosing $C_{-1}$, that is,

$$
C_{-1}=-(-1)^{s(1)} C_{0} m_{-s(1)} \gamma_{1}=-(-1)^{s(1)} \frac{m_{-s(1)}}{\sqrt{m_{0}}} .
$$

Here $\gamma_{1}$ denotes the leading coefficient of $P_{1}$ where $\Psi_{1}(x)=\frac{P_{1}(x)}{x^{p(1)}}$.

Remark 3.6. If we write $\Sigma_{n}(z)=\frac{Q_{n}(z)}{z^{p(n)}}$, with $Q_{n} \in \mathbb{P}_{n-1}, n \geq 1$, we can also obtain

$$
C_{n} Q_{n+1}(z)=(-1)^{s(n)}\left[z^{1-2 s(n)}-\Omega_{n}\right] z^{s(n)} Q_{n}(z)-C_{n-1} z^{s(n-1)+s(n)} Q_{n-1}(z),
$$

$n \geq 0$, with $Q_{0}(z) \equiv 0, Q_{-1}(z) \equiv 1$ and $C_{-1}$ given by (3.18). In case we choose $s(-1)=1$, and since $s(0)=0, Q_{-1}(z)$ should be taken as $\frac{1}{z}$. 
On the other hand, setting $z=y$ in (3.1) further yields the following corollary.

Corollary 3.7 (Confluent formula). Under the same assumptions as in Theorem 3.1, it holds for $n \geq 1$ that

$$
\begin{aligned}
\sum_{k=0}^{n} \Psi_{k}^{2}(x)=C_{n} x^{s(n+1)+s(n)}[ & \Psi_{n+1}^{\prime}(x) \Psi_{n}(x)-\Psi_{n}^{\prime}(x) \Psi_{n+1}(x) \\
& \left.+\frac{s(n+1)-s(n)}{x} \Psi_{n}(x) \Psi_{n+1}(x)\right] .
\end{aligned}
$$

We have already seen that the zeros of $\Psi_{n}$ are distinct and are contained in $(a, b)$. Now, making use of the confluent formula (3.19), a standard argument (see, e.g., [11]) leads to the interlacing property of the zeros of $\Psi_{n+1}$ with those of $\Psi_{n}$. Indeed, one has

Corollary 3.8. For each $n \geq 1$, let $\left\{x_{j, n}\right\}_{j=1}^{n}$ denote the zeros of $\Psi_{n}$ so that $a<x_{1, n}<x_{2, n}<\cdots<x_{n, n}<b$. Then

$$
x_{j, n+1}<x_{j, n}<x_{j+1, n+1}, \quad j=1,2, \ldots, n .
$$

\section{Quadratures}

Let $\phi$ be an SSD and suppose the integral

$$
I_{\phi}(f)=\int_{a}^{b} f(t) d \phi(t)
$$

needs to be approximated by an $n$-point quadrature formula of the form

$$
I_{n}(f)=\sum_{j=1}^{n} A_{j} f\left(x_{j}\right) .
$$

Since we suppose that the moments (1.1) exist, it seems natural that the $n$ distinct nodes $\left\{x_{j}\right\}_{j=1}^{n} \subset(a, b)$ and the coefficients or weights $\left\{A_{j}\right\}_{j=1}^{n} \subset \mathbb{R}$ are to be determined by imposing that $I_{\phi}(L)=I_{n}(L)$ for any L-polynomial $L$ belonging to a linear subspace of $\Delta$ of dimension as large as possible. Thus, starting from the nested sequence of linear subspaces of L-polynomials $\left\{\mathcal{L}_{n}\right\}_{n=0}^{\infty}$ defined above and fixing $n$ distinct points $\left\{x_{j}\right\}_{j=1}^{n}$ on $(a, b)$, it is known that there exists a unique set of quadrature coefficients $\left\{A_{j}\right\}_{j=1}^{n}$ such that

$$
I_{\phi}(L)=I_{n}(L), \forall L \in \mathcal{L}_{n-1}
$$

(see [7]), i.e., the corresponding quadrature rule exactly "integrates" any L-polynomial in $\mathcal{L}_{n-1}$.

On the other hand, let $L_{n}(f ; x)$ be the unique L-polynomial in $\mathcal{L}_{n-1}$ interpolating $f$ at the $n$ distinct nodes $\left\{x_{j}\right\}_{j=1}^{n}$, so that one can write

$$
L_{n}(f ; x)=\sum_{j=1}^{n} f\left(x_{j}\right) L_{j, n}(x)
$$

where $L_{j, n} \in \mathcal{L}_{n-1}$ satisfies $L_{j, n}\left(x_{k}\right)=\delta_{j, k}, 1 \leq j, k \leq n$.

More precisely, if we set

$$
W_{n}(x)=\frac{\left(x-x_{1}\right)\left(x-x_{2}\right) \cdots\left(x-x_{n}\right)}{x^{p(n)}} \in \mathcal{L}_{n},
$$


then

$$
L_{j, n}(x)=\left(\frac{x}{x_{j}}\right)^{s(n)} \frac{W_{n}(x)}{\left(x-x_{j}\right) W_{n}^{\prime}\left(x_{j}\right)}, s(n)=p(n)-p(n-1) .
$$

Now, it can be easily proved that the quadrature formula $I_{n}(f)$ obtained from (4.1) is given by

$$
I_{n}(f)=I_{\phi}\left(L_{n}(f ; \cdot)\right)=\sum_{j=1}^{n} I_{\phi}\left(L_{j, n}\right) f\left(x_{j}\right)
$$

Thus,

$$
A_{j}=I_{\phi}\left(L_{j, n}\right)=\int_{a}^{b} L_{j, n}(t) d \phi(t), j=1,2, \ldots, n .
$$

For this reason, $I_{n}(f)$ given by (4.1) is sometimes called of interpolatory type in $\mathcal{L}_{n-1}$. Next, we will show that choosing the nodes as the zeros of the orthogonal L-polynomial $\psi_{n}$ leads to a much higher accuracy. For this purpose, we will investigate how to construct quadrature rules to be exact in linear subspaces of L-polynomials of the form $\mathcal{L}_{n} \mathcal{L}_{r}$ with $r \geq 0$ (the space consisting of the usual pointwise-defined product of L-polynomials of the two L-spaces). Observe that $\mathcal{L}_{n} \mathcal{L}_{r}=\Delta_{-p(n)-p(r), q(n)+q(r)}$, whose dimension is $n+r+1$, and that in general $\mathcal{L}_{n} \mathcal{L}_{r} \neq \mathcal{L}_{n+r}$. Actually, we are trying to parallel the polynomial situation where exactness is enlarged from $\mathbb{P}_{n-1}$ to $\mathbb{P}_{n+r}=\mathbb{P}_{n} \mathbb{P}_{r}, 0 \leq r \leq n-1$; see 15.

Furthermore $r \leq n-1$, since it can be checked that there cannot exist an $n$-point quadrature formula to be exact in $\mathcal{L}_{n} \mathcal{L}_{n}$. After these considerations, we can prove the following Jacobi-type theorem,

Theorem 4.1. Let $I_{n}(f)=\sum_{j=1}^{n} A_{j} f\left(x_{j}\right)$ be an n-point quadrature rule such that $x_{i} \neq x_{j}$ and $x_{i} \neq 0$. Then, $I_{n}(f)$ is exact in $\mathcal{L}_{n} \mathcal{L}_{r}(0 \leq r \leq n-1)$, if and only if,

(1) $I_{n}(f)$ is exact in $\mathcal{L}_{n-1}$,

$$
\left\langle W_{n}, L\right\rangle_{\phi}=0, \quad \forall L \in \mathcal{L}_{r}, \quad \text { where } \quad W_{n}(x)=\frac{\prod_{j=1}^{n}\left(x-x_{j}\right)}{x^{p(n)}} \in \mathcal{L}_{n}
$$

Proof. See [15] for $p(n)=0$ and 13$]$ for $p(n)=E\left[\frac{n+1}{2}\right]$. For the general case, let $I_{n}(f)$ be exact in $\mathcal{L}_{n} \mathcal{L}_{r}$. Since $\mathcal{L}_{n-1} \subset \mathcal{L}_{n} \mathcal{L}_{r}$, then $I_{n}(f)$ is trivially exact in $\mathcal{L}_{n-1}$. Now, taking into account that

$$
W_{n} L \in \mathcal{L}_{n} \mathcal{L}_{r} \Longleftrightarrow L \in \mathcal{L}_{r},
$$

and because of exactness in $\mathcal{L}_{n} \mathcal{L}_{r}$, one can write

$$
\left\langle W_{n}, L\right\rangle_{\phi}=I_{\phi}\left(W_{n} L\right)=\sum_{j=1}^{n} A_{j} W_{n}\left(x_{j}\right) L\left(x_{j}\right)=0 \quad \text { since } W_{n}\left(x_{k}\right)=0,1 \leq k \leq n .
$$

Conversely, take $R \in \mathcal{L}_{n} \mathcal{L}_{r}$ and let $L_{n}(R ; \cdot)$ denote the unique L-polynomials in $\mathcal{L}_{n-1}$ interpolating $R$ at the nodes $\left\{x_{j}\right\}_{j=1}^{n}$. Since $R-L_{n}(R ; \cdot) \in \mathcal{L}_{n} \mathcal{L}_{r}$, it holds that

$$
R(x)-L_{n}(R ; x)=W_{n}(x) L(x), \quad L \in \mathcal{L}_{r}
$$


Thus,

$$
\begin{aligned}
I_{\phi}(R) & =I_{\phi}\left(L_{n}(R ; \cdot)\right)+I_{\phi}\left(W_{n} L\right) \\
& =I_{\phi}\left(L_{n}(R ; \cdot)\right) \\
& =I_{n}\left(L_{n}(R, \cdot)\right) \\
& =I_{n}(R), \text { since } R\left(x_{j}\right)=L_{n}\left(R, x_{j}\right), \quad j=1,2, \ldots, n .
\end{aligned}
$$

Now the question is, what about the zeros of a L-polynomial $W_{n}$ satisfying (4.5). Since $W_{n} \in \mathcal{L}_{n}$ one can write $W_{n}(x)=\sum_{j=0}^{n} q_{j} \psi_{j}(x),\left\{\psi_{n}\right\}_{n \geq 0}$ being the orthogonal sequence. Thus, by (4.5) one has

$$
W_{n}(x)=\sum_{j=r+1}^{n} a_{j} \psi_{j}(x) \quad \text { with } \quad a_{n}=1 .
$$

Hence, by taking $r=n-1$, the following holds:

Corollary 4.2 (L-orthogonal quadrature). Let $I_{n}(f)=\sum_{j=1}^{n} A_{j, n} f\left(x_{j, n}\right)$ be an npoint quadrature rule with $n$ distinct nodes on $(a, b)$. Then, $I_{n}(L)=I_{\phi}(L), \forall L \in$ $\mathcal{L}_{n} \mathcal{L}_{n-1}$ if and only if

(1) $I_{n}(f)$ is exact in $\mathcal{L}_{n-1}$,

(2) the nodes $\left\{x_{j, n}\right\}_{j=1}^{n}$ are the zeros of the $n$-th orthogonal L-polynomials $\psi_{n}$.

Furthermore, the weights $\left\{A_{j, n}\right\}_{j=1}^{n}$ are positive.

Proof. It only remains to prove that $A_{j, n}>0, j=1,2, \ldots, n$. Considering $L_{j, n} \in$ $\mathcal{L}_{n-1}$ given by,

$$
L_{j, n}(x)=\left(\frac{x}{x_{j, n}}\right)^{s(n)} \frac{\psi_{n}(x)}{\left(x-x_{j, n}\right) \psi_{n}^{\prime}\left(x_{j, n}\right)},
$$

we have

$$
L_{j, n}^{2} \in \mathcal{L}_{n-1} \mathcal{L}_{n-1} \subset \mathcal{L}_{n} \mathcal{L}_{n-1} \quad \text { and } \quad L_{j, n}^{2}\left(x_{k}\right)=\delta_{j, k}
$$

Hence,

$$
I_{\phi}\left(L_{j, n}^{2}\right)=I_{n}\left(L_{j, n}^{2}\right)=A_{j, n}>0, j=1,2, \ldots, n .
$$

Remark 4.3. The quadrature formula $I_{n}(f)$ given in Corollary 4.2 will be called L-orthogonal (see [7]) for the SSD $\phi$ and the "ordering" induced by $\{p(n)\}_{n \geq 0}$. Observe that in general we do not have exactness in $\mathcal{L}_{2 n-1}$ (L-Gaussian formula). The latter happens if and only if the sequence $\{p(n)\}_{n \geq 0}$ satisfies $p(n)+p(n-1)=$ $p(2 n-1)$, because in this case $\mathcal{L}_{n} \mathcal{L}_{n-1}=\mathcal{L}_{2 n-1}$. This is true, for instance, when $p(n)=0$ or $p(n)=E\left[\frac{n+1}{2}\right]$, giving rise to the usual Gaussian formulas and the L-Gaussian formulas studied in [25] (see also [12, 22]). However, $n$-point quadrature rules with nodes on $(a, b)$ and exact in $\mathcal{L}_{2 n-1}$ can also be obtained. In this situation, the nodes are the zeros of the $n$-th orthogonal polynomial with respect to the varying distribution $\frac{d \phi(t)}{t^{p(2 n-1)}}$. This kind of quadratures can be considered as a particular case of those studied by some of the present authors in [6.

Another explicit representation for the coefficients $\left\{A_{j, n}\right\}_{j=1}^{n}$ in the $n$-point Lorthogonal formula can be given as follows: 
Proposition 4.4. Let $\phi$ be an SSD and $\left\{\psi_{n}\right\}_{n \geq 0}$ the orthogonal sequence of $L$ polynomials associated with a nondecreasing sequence $\{p(n)\}_{n \geq 0}$ such that $0 \leq$ $p(n) \leq n$ and $p(n)-p(n-1)=s(n) \in\{0,1\}$ for each $n$. Let $\left\{\sigma_{n}\right\}_{n \geq 0}$ be the corresponding sequence of "associated" L-polynomials to $\left\{\psi_{n}\right\}_{n \geq 0}$ as given by (2.4). Then, it holds that

$$
A_{j, n}=\frac{\sigma_{n}\left(x_{j, n}\right)}{\psi_{n}^{\prime}\left(x_{j, n}\right)}, j=1, \ldots, n,
$$

where $\left\{x_{j, n}\right\}_{j=1}^{n}$ are the zeros of $\psi_{n}$.

Proof. From (4.2)-(4.4) one can write

$$
A_{j, n}=\frac{1}{x_{j, n}^{s(n)} \psi_{n}^{\prime}\left(x_{j, n}\right)} \int_{a}^{b} \frac{t^{s(n)} \psi_{n}(t)}{t-x_{j, n}} d \phi(t) .
$$

As a consequence of the orthogonality conditions,

$$
\int_{a}^{b} \psi_{n}(t) \frac{t^{s(n)} L(t)-x_{j, n}^{s(n)} L\left(x_{j, n}\right)}{t-x_{j, n}} d \phi(t)=0, \quad \forall L \in \mathcal{L}_{n} .
$$

By taking $L \equiv 1$, it follows that

$$
\begin{aligned}
A_{j, n} & =\frac{1}{\psi_{n}^{\prime}\left(x_{j, n}\right)} \int_{a}^{b} \frac{\psi_{n}(t)}{t-x_{j, n}} d \phi(t) \\
& =\frac{1}{\psi_{n}^{\prime}\left(x_{j, n}\right)} \int_{a}^{b} \frac{\psi_{n}(t)-\psi_{n}\left(x_{j, n}\right)}{t-x_{j, n}} d \phi(t)=\frac{\sigma_{n}\left(x_{j, n}\right)}{\psi_{n}^{\prime}\left(x_{j, n}\right)} .
\end{aligned}
$$

Remark 4.5. Setting, $\psi_{n}(x)=\frac{p_{n}(x)}{x^{p(n)}}$ and $\sigma_{n}(x)=\frac{q_{n}(x)}{x^{p(n)}}$ with $p_{n}$ and $q_{n}$ polynomials of degree $n$ and $n-1$, respectively, one can also write

$$
A_{j, n}=\frac{q_{n}\left(x_{j, n}\right)}{p_{n}^{\prime}\left(x_{j, n}\right)}, \quad j=1,2, \ldots, n,
$$

where $\left\{x_{j, n}\right\}_{j=1}^{n}$ are the zeros of $p_{n}\left(\right.$ or $\left.\psi_{n}\right)$.

From these results, the following can be immediately deduced:

Corollary 4.6. Between two consecutive zeros of $\psi_{n}$ there exists at least one zero of $\sigma_{n}$.

Proof. From (4.8),

$$
\frac{\sigma_{n}(z)}{\psi_{n}(z)}=\frac{q_{n}(z)}{p_{n}(z)}=\sum_{j=1}^{n} \frac{A_{j, n}}{z-x_{j, n}}
$$

where $\left\{x_{j, n}\right\}_{j=1}^{n}$ are the zeros of $p_{n}$ (or $\left.\psi_{n}\right)$ and $A_{j, n}>0$. Now, make use of the standard argument saying that a simple partial fraction decomposition of a rational function involving positive coefficients is equivalent to the fact that the zeros of the denominator separate those of the numerator.

Remark 4.7. From the corollary above, one sees that $\sigma_{n}$ has exactly $n-1$ distinct zeros on $(a, b)$.

In the following representation, positivity of the coefficients $\left\{A_{j, n}\right\}_{j=1}^{n}$ is clearly displayed. Indeed, one has (compare with Theorem 3.5 in [9]) 
Theorem 4.8 (L-Christoffel numbers). Let $\left\{\Psi_{n}\right\}_{n \geq 0}$ be the sequence of orthonormal L-polynomials with respect to the SSD $\phi$ associated with the "ordering" induced by a nondecreasing sequence $\{p(n)\}_{n \geq 0}$, such that $0 \leq p(n) \leq n$ and $p(n)-$ $p(n-1)=s(n) \in\{0,1\}$ for each $n$. Let $\left\{x_{j, n}\right\}_{j=1}^{n}$ be the zeros of $\Psi_{n}$ and define the Christoffel function

$$
\mathcal{K}_{n}(x)=\frac{1}{\mathcal{K}_{n}(x, x)}=\frac{1}{\sum_{k=0}^{n} \Psi_{k}^{2}(x)} .
$$

Then, the weights $\left\{A_{j, n}\right\}_{j=1}^{n}$ of the $n$-th L-orthogonal formula are given by

$$
A_{j, n}=\mathcal{K}_{n}\left(x_{j, n}\right), j=1,2, \ldots, n .
$$

Proof. Recalling (4.7) and taking into account that $\Psi_{k}=\gamma_{k} \psi_{k}$, it follows that

$$
A_{j, n}=\frac{1}{x_{j, n}^{s(n)} \Psi_{n}^{\prime}\left(x_{j, n}\right)} \int_{a}^{b} \frac{t^{s(n)} \Psi_{n}(t)}{t-x_{j, n}} d \phi(t) .
$$

Now, consider the Christoffel-Darboux identity, i.e.,

$$
\sum_{k=0}^{n} \Psi_{k}(x) \Psi_{k}(y)=C_{n}\left[\frac{x^{s(n+1)} \Psi_{n+1}(x) y^{s(n)} \Psi_{n}(y)-x^{s(n)} \Psi_{n}(x) y^{s(n+1)} \Psi_{n+1}(y)}{x-y}\right],
$$

where $n=1,2, \ldots$. Now set $y=x_{j, n}$. Then $\left(\Psi_{n}\left(x_{j, n}\right)=0\right)$

$$
\sum_{k=0}^{n-1} \Psi_{k}(x) \Psi_{k}\left(x_{j, n}\right)=-C_{n} \frac{x^{s(n)} \Psi_{n}(x) x_{j, n}^{s(n+1)} \Psi_{n+1}\left(x_{j, n}\right)}{x-x_{j, n}} .
$$

Since $\int_{a}^{b} \Psi_{i}(t) d \phi(t)=\delta_{i, 0}$, it follows that

$$
1=-C_{n} x_{j, n}^{s(n+1)} \Psi_{n+1}\left(x_{j, n}\right) \int_{a}^{b} \frac{t^{s(n)} \Psi_{n}(t)}{t-x_{j, n}} d \phi(t) .
$$

Thus,

$$
A_{j, n}=-\frac{1}{C_{n} x_{j, n}^{s(n)+s(n+1)} \Psi_{n}^{\prime}\left(x_{j, n}\right) \Psi_{n+1}\left(x_{j, n}\right)} .
$$

Finally, by Corollary 3.7 .

$$
\sum_{k=0}^{n-1} \Psi_{k}^{2}\left(x_{j, n}\right)=-C_{n} x_{j, n}^{s(n)+s(n+1)} \Psi_{n+1}\left(x_{j, n}\right) \Psi_{n}^{\prime}\left(x_{j, n}\right) .
$$

So, by the two last equalities the proof is concluded.

Remark 4.9. Making use of Corollary 3.7, one can also deduce the following as an alternative to (4.9):

$$
A_{j, n}=\frac{1}{C_{n-1} x_{j, n}^{s(n)+s(n-1)} \Psi_{n}^{\prime}\left(x_{j, n}\right) \Psi_{n-1}\left(x_{j, n}\right)}, j=1, \ldots, n .
$$

Next we can work out an extremal property for the orthogonal Laurent polynomials where a nice interpretation of extremal zeros is now given (see, e.g., [11] for the polynomial situation).

Proposition 4.10 (Chebyshev). Let $\left\{x_{j, n}\right\}_{j=1}^{n}$ be the zeros of the $n$-th orthogonal $L$-polynomial $\psi_{n}$ for the SSD $\phi$ so that $x_{1, n}<x_{2, n}<\cdots<x_{n, n}$. Then, 
(1) $\max _{L \in \mathcal{L}_{n-1}} \frac{\int_{a}^{b} t^{(-1)^{s(n)}} L^{2}(t) d \phi(t)}{\int_{a}^{b} L^{2}(t) d \phi(t)}=\frac{s(n)}{x_{1, n}}+(1-s(n)) x_{n, n}$,

(2) $\min _{L \in \mathcal{L}_{n-1}} \frac{\int_{a}^{b} t^{(-1)^{s(n)}} L^{2}(t) d \phi(t)}{\int_{a}^{b} L^{2}(t) d \phi(t)}=\frac{s(n)}{x_{n, n}}+(1-s(n)) x_{1, n}$,

where $s(n)=p(n)-p(n-1) \in\{0,1\}$ and $\{p(n)\}_{n \geq 0}$ denote the sequence generating $\left\{\mathcal{L}_{n}\right\}_{n \geq 0}$.

Proof. Let $I_{n}(f)=\sum_{j=1}^{n} A_{j, n} f\left(x_{j, n}\right)$ be the $n$-th L-orthogonal formula for $d \phi$. Now, for any $L \in \mathcal{L}_{n-1}, L^{2} \in \mathcal{L}_{n-1} \mathcal{L}_{n-1} \subset \mathcal{L}_{n} \mathcal{L}_{n-1}$. We conclude also that $t^{(-1)^{s(n)}} L^{2} \in \mathcal{L}_{n} \mathcal{L}_{n-1}$.

As a consequence of the exactness of $I_{n}(f)$ in $\mathcal{L}_{n} \mathcal{L}_{n-1}$, one has

$$
\begin{aligned}
\int_{a}^{b} t^{(-1)^{s(n)}} L^{2}(t) d \phi(t) & =\sum_{j=1}^{n} A_{j, n} x_{j, n}^{(-1)^{s(n)}} L^{2}\left(x_{j, n}\right), \\
\int_{a}^{b} L^{2}(t) d \phi(t) & =\sum_{j=1}^{n} A_{j, n} L^{2}\left(x_{j, n}\right) .
\end{aligned}
$$

Since $x_{j, n}, A_{j, n}$ are positive, it then follows that

$$
\frac{s(n)}{x_{n, n}}+(1-s(n)) x_{1, n} \leq \frac{\int_{a}^{b} t^{(-1)^{s(n)}} L^{2}(t) d \phi(t)}{\int_{a}^{b} L^{2}(t) d \phi(t)} \leq \frac{s(n)}{x_{1, n}}+(1-s(n)) x_{n, n} .
$$

Now the lower bound is reached by

$$
L=s(n) L_{n, n}+(1-s(n)) L_{1, n},
$$

while the upper one is reached by

$$
L=s(n) L_{1, n}+(1-s(n)) L_{n, n} .
$$

Here $L_{j, n}, 1 \leq j \leq n$ are given by (4.2)

We conclude this section giving an error expression for the $n$-th L-orthogonal formula. This expression appears just by rewriting Theorem 2.3 in 4 in terms of orthogonal Laurent polynomials. Indeed, let $\psi_{n}$ be the $n$-th orthogonal L-polynomial normalized as in Section 2 and let $f$ be a function Riemann-Stieltjes integrable with respect to $\phi$ such that $f^{(2 n)}(t)$ is continuous for $a \leq t \leq b$. If we define $F(t)=\frac{f(t)}{t^{p(n)+p(n-1)}}$, then

$$
I_{\phi}(F)-I_{n}(F)=\frac{f^{(2 n)}(\mu)}{(2 n) !} \int_{a}^{b} t^{s(n)} \psi_{n}^{2}(t) d \phi(t)
$$

where $p(n)=s(n)+p(n-1)$ and $\mu \in(a, b)$. Here $I_{n}(F)$ denotes the $n$-th Lorthogonal formula for $d \phi$, so that the above expression of the error can be helpful, when dealing with smooth enough integrands and provided that we can estimate the higher order derivatives. In the following section, an alternative error expression will be given for a class of more restrictive integrands.

Remark 4.11. Once again, it should be noted that for $p(n)=0(s(n)=0)$ from the results given up to this section, known properties and results for orthogonal polynomials can be obtained. 


\section{Two-point Padé approximation}

As is known, the so-called multipoint Padé approximants arise as a natural generalization of the classical (one-point) Padé approximants when information about the function to be approximated is given in terms of Taylor series or asymptotic expansions around more than one point. When expansions around the origin and infinity are considered, two-point Padé approximants (2PA) appear. They were first introduced in the context of different physical problems [2] and of continued fractions [24.

For a definition of those approximants and in order to make the paper selfcontained we will start as in [10] (compare with 28] essentially inspired by 29]). Given two formal power series

$$
f_{0}(z)=\sum_{k=0}^{\infty} f_{k}^{*} z^{k}, \quad z \rightarrow 0, \quad \text { and } \quad f_{\infty}(z)=\sum_{k=1}^{\infty} f_{k} \frac{1}{z^{k}}, z \rightarrow \infty,
$$

and two nonnegative integers $k$ and $n(0 \leq k \leq 2 n)$, polynomials $P_{k, n}\left(P_{k, n} \not \equiv 0\right)$ and $Q_{k, n}$ of degree $n$ and $n-1$, respectively, are to be found such that

$$
\begin{aligned}
P_{k, n}(z) f_{0}(z)-Q_{k, n}(z) & =d_{k, n}^{*} z^{k}+d_{k+1, n}^{*} z^{k+1}+\cdots=\mathcal{O}\left(z^{k}\right), \quad z \rightarrow 0 \\
P_{k, n}(z) f_{\infty}(z)-Q_{k, n}(z) & =\frac{d_{n+1-k, n}}{z^{n+1-k}}+\frac{d_{n+2-k, n}}{z^{n+2-k}}+\cdots=\mathcal{O}\left(\frac{1}{z^{n+1-k}}\right), \quad z \rightarrow \infty .
\end{aligned}
$$

Clearly, when $P_{k, n}$ has exact degree $n$ and $P_{k, n}(0) \neq 0$, from (5.2) one has

$$
\begin{aligned}
f_{0}(z)-\frac{Q_{k, n}(z)}{P_{k, n}(z)} & =\mathcal{O}\left(z^{k}\right), z \rightarrow 0, \\
f_{\infty}(z)-\frac{Q_{k, n}(z)}{P_{k, n}(z)} & =\mathcal{O}\left(\frac{1}{z^{2 n+1-k}}\right), \quad z \rightarrow \infty .
\end{aligned}
$$

Furthermore, any solution $\left(P_{k, n}, Q_{k, n}\right)$ of (5.2) defines a unique rational function $\frac{Q_{k, n}}{P_{k, n}}$ that is said to be a $[k / n]-2 \mathrm{PA}$ to $\left(f_{0}, f_{\infty}\right)$ in the "weak sense", while if conditions (5.3) are satisfied, it is said to be a $[k / n]-2 \mathrm{PA}$ in the "strong sense".

In both cases, it will be denoted as $[k / n]_{f_{0}, f_{\infty}}$. Observe that the parameter $k$ represents the number of coefficients matched in $f_{0}$.

An important class of functions admitting expansions of the form (5.1) is the Cauchy transform of a distribution $\phi$ on $(a, b)$, i.e., $\int_{a}^{b} \frac{d \phi(t)}{z-t}$. When $\phi$ is an SSD, it will be called its Stieltjes transform and we will write

$$
F_{\phi}(z)=\int_{a}^{b} \frac{d \phi(t)}{z-t}, z \notin[a, b], 0 \leq a<b \leq \infty .
$$

Thus, if $m_{k}=\int_{a}^{b} t^{k} d \phi(t), k=0, \pm 1, \pm 2, \ldots$, are the moments of $d \phi$, then $F_{\phi}$ admits the formal expansions (convergent or asymptotic; see [26] for details)

$$
F_{0}(z)=-\sum_{k=0}^{\infty} m_{-(k+1)} z^{k}, z \rightarrow 0, \quad \text { and } \quad F_{\infty}(z)=\sum_{k=0}^{\infty} m_{k} \frac{1}{z^{k+1}}, z \rightarrow \infty .
$$

In this case, the $[k / n]-2 \mathrm{PA}$ to the pair $\left(F_{0}, F_{\infty}\right)$ or to the function $F_{\phi}$ will be denoted by $[k / n]_{F_{\phi}}$. Approximating functions of the form (5.4) by rational functions making use of (5.5) has become an important task in the field of special functions and applications (see [22] and also [23]). The main aim of this section is bringing 
together some known results and properties about two-point Padé approximants to $F_{\phi}$ by means of the theory of orthogonal Laurent polynomials and L-orthogonal formulas. The results given in [9] will now be completed, since there the setting reduces to sequences of nonnegative integers $\{p(n)\}_{n \geq 0}$ satisfying $p(n)+p(n-1)=$ $p(2 n-1)$ for each $n$. So, as in the previous section, let $\{p(n)\}_{n \geq 0}$ be a nondecreasing sequence of nonnegative integers such that $0 \leq p(n) \leq n$ and $p(n)-p(n-1)=$ $s(n) \in\{0,1\}$ for each $n$. Let

$$
I_{n}(f)=\sum_{j=1}^{n} A_{j, n} f\left(x_{j, n}\right)
$$

be the $n$-th L-orthogonal formula for the SSD $\phi$ associated with the "ordering" induced by $\{p(n)\}_{n \geq 0}$.

For $z \notin[a, b]$, an approximation for (5.4) can be given by using this $n$-th Lorthogonal formula. Indeed, one has by 4.6)

$$
F_{\phi}(z)=I_{\phi}\left(\frac{1}{z-\cdot}\right) \approx I_{n}\left(\frac{1}{z-\cdot}\right)=\sum_{j=1}^{n} \frac{A_{j, n}}{z-x_{j, n}}=\frac{\sigma_{n}(z)}{\psi_{n}(z)} .
$$

On the other hand, since $I_{n}(f)$ is exact in $\mathcal{L}_{n} \mathcal{L}_{n-1}$, it can be checked that the following hold:

$$
\begin{aligned}
F_{0}(z)-\frac{\sigma_{n}(z)}{\psi_{n}(z)} & =\mathcal{O}\left(z^{p(n)+p(n-1)}\right), \quad z \rightarrow 0, \\
F_{\infty}(z)-\frac{\sigma_{n}(z)}{\psi_{n}(z)} & =\mathcal{O}\left(\frac{1}{z^{2 n+1-p(n)-p(n-1)}}\right), \quad z \rightarrow \infty .
\end{aligned}
$$

In other words, we have proved the following proposition.

Proposition 5.1. Let $\left\{\psi_{n}\right\}_{n \geq 0}$ be a sequence of orthogonal L-polynomials for the SSD $\phi$ associated with the "ordering" induced by a nondecreasing sequence $\{p(n)\}_{n \geq 0}$ such that $0 \leq p(n) \leq n$ and $p(n)-p(n-1)=s(n) \in\{0,1\}$ for each $n$. Let $\left\{\sigma_{n}\right\}_{n \geq 0}$ be the corresponding sequence of associated L-polynomials. Then,

$$
[p(n)+p(n-1) / n]_{F_{\phi}}=\frac{\sigma_{n}}{\psi_{n}} .
$$

Moreover, by (4.3) it also holds that

$$
[p(n)+p(n-1) / n]_{F_{\phi}}(z)=I_{n}\left(\frac{1}{z-\cdot}\right)=I_{\phi}\left(L_{n}\left(\frac{1}{z-\cdot} ; \cdot\right)\right)
$$

where $L_{n}\left(\frac{1}{z-.} ; \cdot\right)$ represents the interpolating L-polynomial in $\mathcal{L}_{n-1}$ for the function $f(x)=f(x, z)=\frac{1}{z-x}(z$ a parameter $)$ at the zeros of $\psi_{n}$.

It can be checked that

$$
L_{n}\left(\frac{1}{z-\cdot} ; x\right)=\frac{1}{z-x}\left(1-\left(\frac{x}{z}\right)^{s(n)} \frac{\psi_{n}(x)}{\psi_{n}(z)}\right), p(n)=s(n)+p(n-1) .
$$

Hence,

$$
[p(n)+p(n-1) / n]_{F_{\phi}}(z)=\int_{a}^{b}\left(1-\left(\frac{t}{z}\right)^{s(n)} \frac{\psi_{n}(t)}{\psi_{n}(z)}\right) \frac{d \phi(t)}{z-t} .
$$

Set $E_{n}(z)=F_{\phi}(z)-[p(n)+p(n-1) / n]_{F_{\phi}}(z)$. Then from (5.7) one can deduce the following integral representation for the error $E_{n}$ : 
Proposition 5.2. Let $\phi$ be an SSD and $\{p(n)\}_{n \geq 0}$ a nondecreasing sequence of nonnegative integers such that $0 \leq p(n) \leq n$ and $p(n)-p(n-1)=s(n) \in\{0,1\}$ for each $n$. Then, $\forall z \in \mathbb{C} \backslash[a, b]$

$$
E_{n}(z)=\frac{1}{z^{s(n)} \psi_{n}(z)} \int_{a}^{b} \frac{t^{s(n)} \psi_{n}(t)}{z-t} d \phi(t)=\frac{1}{z^{s(n)} \psi_{n}^{2}(z)} \int_{a}^{b} \frac{t^{s(n)} \psi_{n}^{2}(t)}{z-t} d \phi(t) .
$$

Proof. By (5.7) it follows that

$$
[p(n)+p(n-1) / n]_{F_{\phi}}(z)=F_{\phi}(z)-\frac{1}{z^{s(n)} \psi_{n}(z)} \int_{a}^{b} \frac{t^{s(n)} \psi_{n}(t)}{z-t} d \phi(t) .
$$

Now, it should be taken into account that the function in the variable $x$, $\left(\frac{x}{z}\right)^{s(n)} \frac{\psi_{n}(z)-\psi_{n}(x)}{z-x}$, belongs to $\mathcal{L}_{n-1}$. Therefore

$$
\int_{a}^{b} \psi_{n}(t)\left[\left(\frac{t}{z}\right)^{s(n)} \frac{\psi_{n}(z)-\psi_{n}(t)}{z-t}\right] d \phi(t)=0 .
$$

Thus, from this fact and (5.9) the proof can be concluded.

Remark 5.3. Setting $\psi_{n}(x)=\frac{p_{n}(x)}{x^{p(n)}}, p_{n} \in \mathbb{P}_{n}$, we can obtain from (5.8) the known expression for the error in $2 \mathrm{PA}$ (see, e.g., [27]), in terms of the denominator $p_{n}$ of the approximant. Indeed, one has

$$
E_{n}(z)=\frac{z^{p(n)+p(n-1)}}{p_{n}^{2}(z)} \int_{a}^{b} \frac{p_{n}^{2}(t)}{z-t} \frac{d \phi(t)}{t^{p(n)+p(n-1)}} .
$$

On the other hand, when $p(n)=0, p_{n}$ represents the $n$-th monic orthogonal polynomial with respect to $d \phi$ and the above formula becomes

$$
E_{n}(z)=\frac{1}{p_{n}^{2}(z)} \int_{a}^{b} \frac{p_{n}^{2}(t)}{z-t} d \phi(t)
$$

which represents the error of the $[n-1 / n]$ one-point Padé approximant at infinity to $F_{\phi}$.

The forthcoming results are mainly oriented to emphasize the close connection between two-point Padé approximants and L-orthogonal formulas similar to the known relation between one-point Padé approximants at infinity and classical Gaussian formulas. We first have

Theorem 5.4. Let $\phi$ be an $S S D$ with support $[0, \infty)$ and $\{p(n)\}_{n \geq 0}$ a nondecreasing sequence of nonnegative integers such that $0 \leq p(n) \leq n$ and $p(n)-p(n-1)=$ $s(n) \in\{0,1\}$ for each $n$. Let $\mathcal{R}(\phi)$ the set of Riemann-Stieltjes integrable functions with respect to $d \phi$ such that $\lim _{x \rightarrow \infty} f(x)$ exists. Then, the following two statements are equivalent.

(i) $\lim _{n \rightarrow \infty} I_{n}(f)=I_{\phi}(f), \forall f \in \mathcal{R}(\phi)$, where $\left\{I_{n}(f)\right\}_{n \geq 0}$ is the sequence of L-orthogonal formulas for $d \phi$ associated with the ordering induced by $\{p(n)\}_{n \geq 0}$.

(ii) $\lim _{n \rightarrow \infty}[p(n)+p(n-1) / n]_{F_{\phi}}(z)=F_{\phi}(z)$ uniformly on any compact set $K \subset \mathbb{C} \backslash[0, \infty)$.

Proof. (i) $\Longrightarrow$ (ii). It follows by using standard arguments on the normal family $\left\{[p(n)+p(n-1) / n]_{F_{\phi}}\right\}_{n \geq 0}$ and the Stieltjes-Vitali Theorem [20]. 
(ii) $\Longrightarrow$ (i). Proceed as in [4, Theorem 5.3] to obtain the conclusion for a continuous functions $f$ in $[0, \infty)$ such that $\lim _{x \rightarrow \infty} f(x)$ exists, and after that, proceed as in [21, Lemma 2.7].

Thus, by 27, we can deduce in a straightforward way the following (see also [7]):

Corollary 5.5. Let $\phi$ be an $S S D$ on $[0, \infty)$ and let $\left\{m_{k}\right\}_{k \in \mathbb{Z}}$ be its corresponding sequence of moments, i.e., $m_{k}=\int_{0}^{\infty} t^{k} d \phi(t), k=0, \pm 1, \pm 2, \ldots$ Let $\{p(n)\}_{n \geq 0}$ be a nondecreasing sequence of nonnegative integers such that $0 \leq p(n) \leq n$ and $p(n)-p(n-1)=s(n) \in\{0,1\}$ for each $n$. Assume that either

$$
\lim _{n \rightarrow \infty} n-p(n)=\infty \quad \text { and } \quad \sum_{k=1}^{\infty} m_{k}^{-\frac{1}{2 k}}=\infty
$$

or

$$
\lim _{n \rightarrow \infty} p(n)=\infty \quad \text { and } \quad \sum_{k=1}^{\infty} m_{-k}^{-\frac{1}{2 k}}=\infty
$$

hold.

Let $\left\{I_{n}(f)\right\}_{n \geq 0}$ be the sequence of L-orthogonal formulas for $d \phi$ associated with the "ordering" induced by $\{p(n)\}_{n \geq 0}$. Then,

$$
\lim _{n \rightarrow \infty} I_{n}(f)=I_{\phi}(f)
$$

for any function $f$ in $\mathcal{R}(\phi)$.

Concerning analytic integrands, we have the following:

Theorem 5.6. Let $\phi$ be an SSD on $[0, \infty)$ and let $\{p(n)\}_{n \geq 0}$ be a nondecreasing sequence of nonnegative integers such that $0 \leq p(n) \leq n$ and $p(n)-p(n-1)=$ $s(n) \in\{0,1\}$ for each $n$. Assume $f$ is an analytic function in a simply connected domain $G \subset \hat{\mathbb{C}}$ (extended complex plane) which contains the half-line in its interior. Let $I_{n}(f)$ be the $n$-th L-orthogonal formula for d $\phi$ associated with the "ordering" induced by $\{p(n)\}_{n \geq 0}$. Then

$$
I_{\phi}(f)-I_{n}(f)=\frac{1}{2 \pi i} \oint_{\Gamma}\left[F_{\phi}(w)-[p(n)+p(n-1) / n]_{F_{\phi}}(w)\right] f(w) d w,
$$

where $\Gamma$ is any Jordan curve which contains $\mathbb{C} \backslash G$ in its interior and the half-line in its exterior.

Proof. Since $1 \in \mathcal{L}_{n}$ for each $n=0,1, \ldots$, then $I_{\phi}(1)=I_{n}(1)$ and we can assume without loss of generality that $f(\infty)=0$.

Now, by Cauchy's formula for the exterior of $\Gamma$ in $\widehat{\mathbb{C}}$, we have that for $x \in[0, \infty]$

$$
f(x)=\frac{1}{2 \pi i} \oint_{\Gamma} \frac{f(w)}{w-x} d w .
$$


Next, we can prove our theorem following standard arguments. Indeed, one has

$$
\begin{aligned}
I_{\phi}(f) & =\int_{0}^{\infty} f(t) d \phi(t) \\
& =\int_{0}^{\infty}\left(\frac{1}{2 \pi i} \oint_{\Gamma} \frac{f(w)}{w-t} d w\right) d \phi(t) \\
& =\frac{1}{2 \pi i} \oint_{\Gamma} f(w)\left(\int_{0}^{\infty} \frac{d \phi(t)}{w-t}\right) d w \\
& =\frac{1}{2 \pi i} \oint_{\Gamma} f(w) F_{\phi}(w) d w .
\end{aligned}
$$

Here, we have used the Fubini theorem. In a similar way, it also follows that

$$
\begin{aligned}
I_{n}(f) & =\sum_{k=1}^{n} A_{j, n} f\left(x_{j, n}\right) \\
& =\sum_{j=1}^{n} A_{j, n} \frac{1}{2 \pi i} \oint_{\Gamma} \frac{f(w)}{w-x_{j, n}} d w \\
& =\frac{1}{2 \pi i} \oint_{\Gamma} f(w) \sum_{j=1}^{n} \frac{A_{j, n}}{w-x_{j, n}} d w \\
& =\frac{1}{2 \pi i} \oint_{\Gamma} f(w)[p(n)+p(n-1) / n]_{F_{\phi}}(w) d w .
\end{aligned}
$$

Thus, by (5.11) $-(5.12)$ the proof follows.

From the theorem above, one can say, roughly speaking, that when dealing with analytic integrands the error for the L-orthogonal formula is "controlled" by the error of the 2PA. In fact, from (5.8) and (5.10), we get the following corollary.

Corollary 5.7. Under the same assumptions as in Theorem 5.6, it holds that

$$
\left|I_{\phi}(f)-I_{n}(f)\right| \leq \frac{C(f, \Gamma)}{\inf _{z \in \Gamma}\left\{|z|^{s(n)}\left|\psi_{n}(z)\right|^{2}\right\}} \int_{0}^{\infty} t^{s(n)} \psi_{n}^{2}(t) d \phi(t),
$$

with $C(f, \Gamma)$ a positive constant depending on $f$ and $\Gamma$.

Remark 5.8. Further results on convergence of the L-orthogonal formula can be deduced from [6] and [7]. Concerning convergence of 2PA's see [16, 27.

\section{NumericAl RESUlts}

In this section we present several numerical experiments in order to illustrate the interest of the quadrature formulas studied in Section 4 of this paper. On computing these formulas, their connection with two-point Padé approximation introduced in Section 5 will also be displayed.

On the other hand, the role played by the sequence $\{p(n)\}_{n \geq 0}$ giving rise to the "ordering" on the space of L-polynomials will also be emphasized by numerically showing how an appropriate selection of $\{p(n)\}_{n \geq 0}$ can be intimately related to the singularities of the integrand.

As far as we know, examples dealing with $\{p(n)\}_{n \geq 0}$ different from $p(n)=$ 0 (Gauss-Christoffel formulas) and $p(n)=E\left[\frac{n+1}{2}\right]$ or $p(n)=E\left[\frac{n}{2}\right]$ (L-Gaussian formulas, see [6, 17]) have never been considered before. 
Thus, we will be concerned with the estimation of integrals of the form

$$
I(f)=\int_{a}^{b} f(t) d t, \quad 0 \leq a<b \leq \infty
$$

where $f$ may present singularities at the origin and/or infinity. So, it seems natural to look for quadrature formulas exactly integrating L-polynomials like the L-orthogonal formulas studied in this paper. Three different situations are going to be considered depending on the relative position of the singularities of $f$ with respect to the interval $[a, b]$. We will first assume that $0<a<b<\infty$ and that $t=0$ is a possible singularity of $f$. Secondly we will be concerned with functions exhibiting singularities at $t=0$ but now with $[a, b]=[0, b](0<b<\infty)$. Finally, the case $[a, b]=[0, \infty)$ will be considered so that both the origin and infinity may be singularities of $f$.

For this purpose, and as a general rule, (6.1) will be written as

$$
I(f)=I_{\beta}(g)=\int_{a}^{b} g(t) \beta(t) d t \quad \text { and } \quad I(f)=I_{\omega}(h)=\int_{a}^{b} h(t) \omega(t) d t,
$$

where $d \phi(t)=\beta(t) d t$ is a classical distribution on $(a, b)$ (actually $\beta$ is a weight function) and $d \phi(t)=\omega(t) d t$ is an SSD. Under these conditions, $I_{\beta}(g)$ will be approximated by the $n$-point Gaussian formula for $\beta$ denoted by

$$
J_{n}(g)=\sum_{j=1}^{n} B_{j, n} g\left(t_{j, n}\right)
$$

where $\left\{t_{j, n}\right\}_{j=1}^{n}$ are the zeros of the $n$-th orthogonal polynomial with respect to $\beta$ and $\left\{B_{j, n}\right\}_{j=1}^{n}$ are the corresponding $n$-th Christoffel numbers. The computation of (6.2) has been carried out making use of standard software.

On the other hand, $I_{\omega}(h)$ will be estimated by means of an $n$-point L-orthogonal formula for $\omega$, according to different selections of $\{p(n)\}_{n \geq 0}$. These formulas will be written as

$$
I_{n}(h)=\sum_{j=1}^{n} A_{j, n} h\left(x_{j, n}\right) .
$$

The computation of (6.3) has been made by exploiting the connection with 2PA. Thus, starting from the moments $m_{k}=\int_{a}^{b} t^{k} d \phi(t), k=0, \pm 1, \pm 2, \ldots$, we compute for $k(n)=p(n)+p(n-1)$ the $[k(n) / n]-2 \mathrm{PA}$ to the pair $\left(F_{0}, F_{\infty}\right)$ given by (5.5). By setting $[k(n) / n]_{F_{0}, F_{\infty}}=\frac{Q_{n}}{P_{n}}$, one knows that the nodes $\left\{x_{j, n}\right\}_{j=1}^{n}$ in (6.3) are the zeros of $P_{n}$ and that the weights $\left\{A_{j, n}\right\}_{j=1}^{n}$ are given by $A_{j, n}=\frac{Q_{n}\left(x_{j, n}\right)}{P_{n}^{\prime}\left(x_{j, n}\right)}, j=$ $1,2, \ldots, n$, as pointed out in Section 5 .

All the computations have been performed on a Pentium IV workstation using David H. Bailey's ARPREC arbitrary precision package [1. Some complementary computations were made using Maple1 1

The integrands $f$ or, equivalently, $g$ and $h$ have been chosen so that all the involved quadrature processes are convergent.

Furthermore, as for the location of the possible singularities, we will start with an essential singularity at the origin. Then, this type of singularity will be gradually weakened passing through a branch point to a pole and finally introducing another

\footnotetext{
${ }^{1}$ Maple is a trademark of Waterloo Maple Inc.
} 
singularity at a point beyond the right end of the integration interval, in particular at infinity.

In Figures 13, the absolute error for the L-orthogonal and the Gaussian formulas for different values of $n$ and several choices of $\{p(n)\}_{n \geq 0}$ are shown. In the legends, the letter "a" represents the absolute error for $p(n)=E\left[\frac{n+1}{2}\right]$, the letter "b" for $p(n)=E[\sqrt{n}]$, the letter "c" for $p(n)=n-E[\sqrt{n}]$, the letter "d" for $p(n)=$ $E[\log (n)]$, the letter "e" for $p(n)=n-E[\log (n)]$, the letter "f" for $p(n)=n$, the letter "g" for $p(n)=0$, of an $n$-point L-orthogonal formula associated with the SSD $d \phi(t)=\omega(t) d t$. Observe that $p(n)=0$ gives rise to the Gaussian formula for $\omega$. On the other hand, in Figure 2 the letters "le" in the legend denote the absolute error of the $n$-th Gauss-Legendre formula, i.e., $\beta \equiv 1$, and in Figure 3 the letters "lg" in the legend denote the corresponding one for the $n$-th generalized Gauss-Laguerre formula with weight $\beta(t)=\frac{e^{-\frac{t}{2}}}{\sqrt{t}}$.
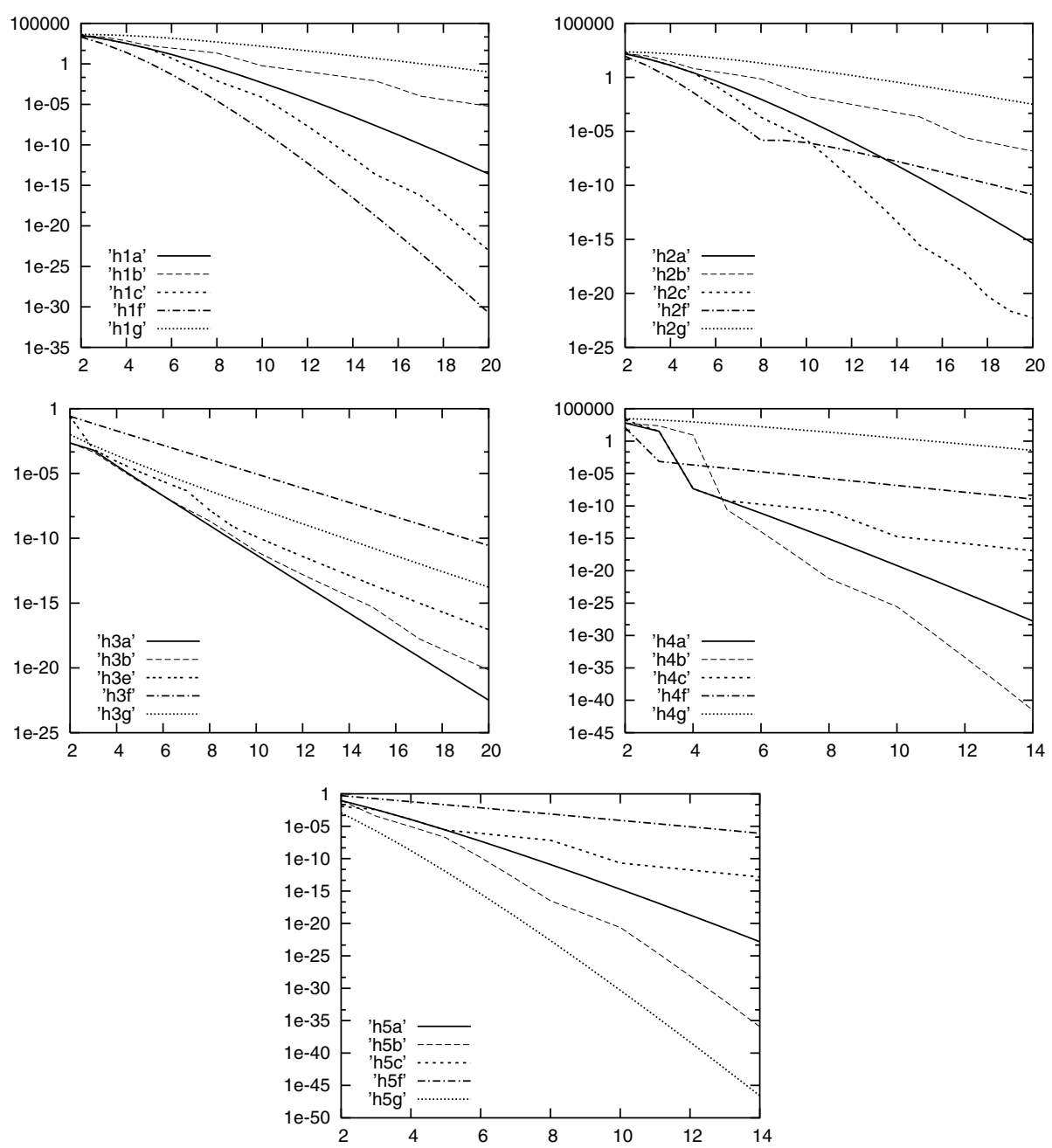

FiguRE 1. Errors as a function of $n$ for Example 6.1 
Example 6.1. Here we consider integrals of the form

$$
\int_{0.1}^{1} f(t) d t
$$

In this case we set $g=h=\frac{f}{\beta}$ where

$$
\beta(t)=\omega(t)=\frac{1}{\sqrt{(t-0.1)(1-t)}},
$$

with the following integrands:

\begin{tabular}{|c|c|}
\hline$i$ & $h_{i}(t)$ \\
\hline 1 & $\exp \left(\frac{1}{t}\right)$ \\
2 & $\exp \left(\frac{1}{t}\right) \sin \left(\frac{t}{2}\right)$ \\
3 & $\exp (\sqrt{t})$ \\
4 & $\frac{\exp (t)}{t^{4}}$ \\
5 & $\exp (t)$ \\
\hline
\end{tabular}

The graphics of the absolute error appear in Figure 1

Example 6.2. Now we will deal with integrals of the form

$$
\int_{0}^{1} f(t) d t
$$

according to the following decomposition: $g=f$ and $\beta \equiv 1$, while $\omega(t)=e^{-\frac{1}{t}}$ and

$$
h(t)=\frac{f(t)}{\omega(t)}=e^{\frac{1}{t}} f(t) .
$$

Numerical experiments were performed by using the following integrands:

\begin{tabular}{|c|c|}
\hline$i$ & $h_{i}(t)$ \\
\hline 1 & $\exp \left(-\frac{5}{3} \frac{\log (t)}{\sqrt{t}}\right)$ \\
2 & $\exp \left(-\frac{\log (t)}{\sqrt{t}}\right) \log (t)$ \\
3 & $\exp \left(\frac{1}{\sqrt{t}}\right) \log (t)$ \\
4 & $\frac{1}{t \sqrt[4]{t^{3}}} \exp \left(\frac{1}{t-\frac{5}{4}}\right)$ \\
5 & $\exp (2 t)$ \\
\hline
\end{tabular}

The corresponding absolute errors are displayed in Figure 2.

Example 6.3. In this example, we shall be concerned with integrals of the form

$$
\int_{0}^{\infty} f(t) d t
$$

so that singularities can appear both at the origin and at infinity. In this case we have $g(t)=f(t) \sqrt{t} e^{\frac{t}{2}}$ and $\beta(t)=\frac{e^{-\frac{t}{2}}}{\sqrt{t}}$, while

$$
\omega(t)=\frac{e^{-\frac{1}{2}\left(t+\frac{1}{t}\right)}}{\sqrt{t}}
$$



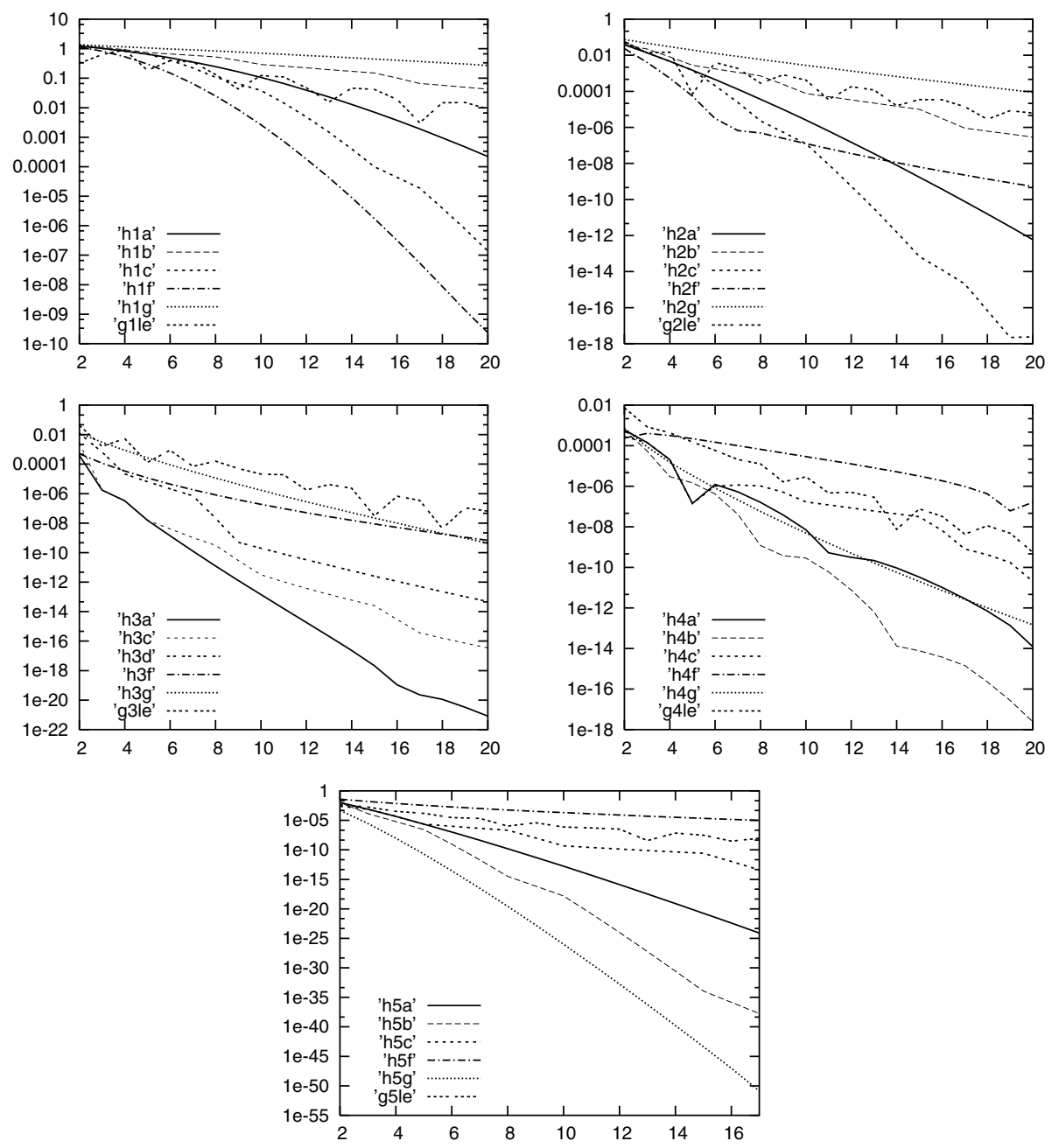

FiguRE 2. Errors as a function of $n$ for Example 6.2

and $h=\frac{f}{\omega}$. The graphics in Figure 3 represent the absolute error for the following functions:

\begin{tabular}{|c|c|}
\hline $\mathrm{i}$ & $h_{i}$ \\
\hline 1 & $\exp \left(\frac{1}{3 t}\right)$ \\
2 & $\exp \left(\frac{1}{3 t}\right) \frac{\log (t)}{t}$ \\
3 & $\frac{\log (t)}{t}$ \\
4 & $\frac{\exp \left(\frac{t}{3}\right)}{\sqrt[8]{t}}$ \\
5 & $\exp \left(\frac{t}{3}\right)$ \\
\hline
\end{tabular}



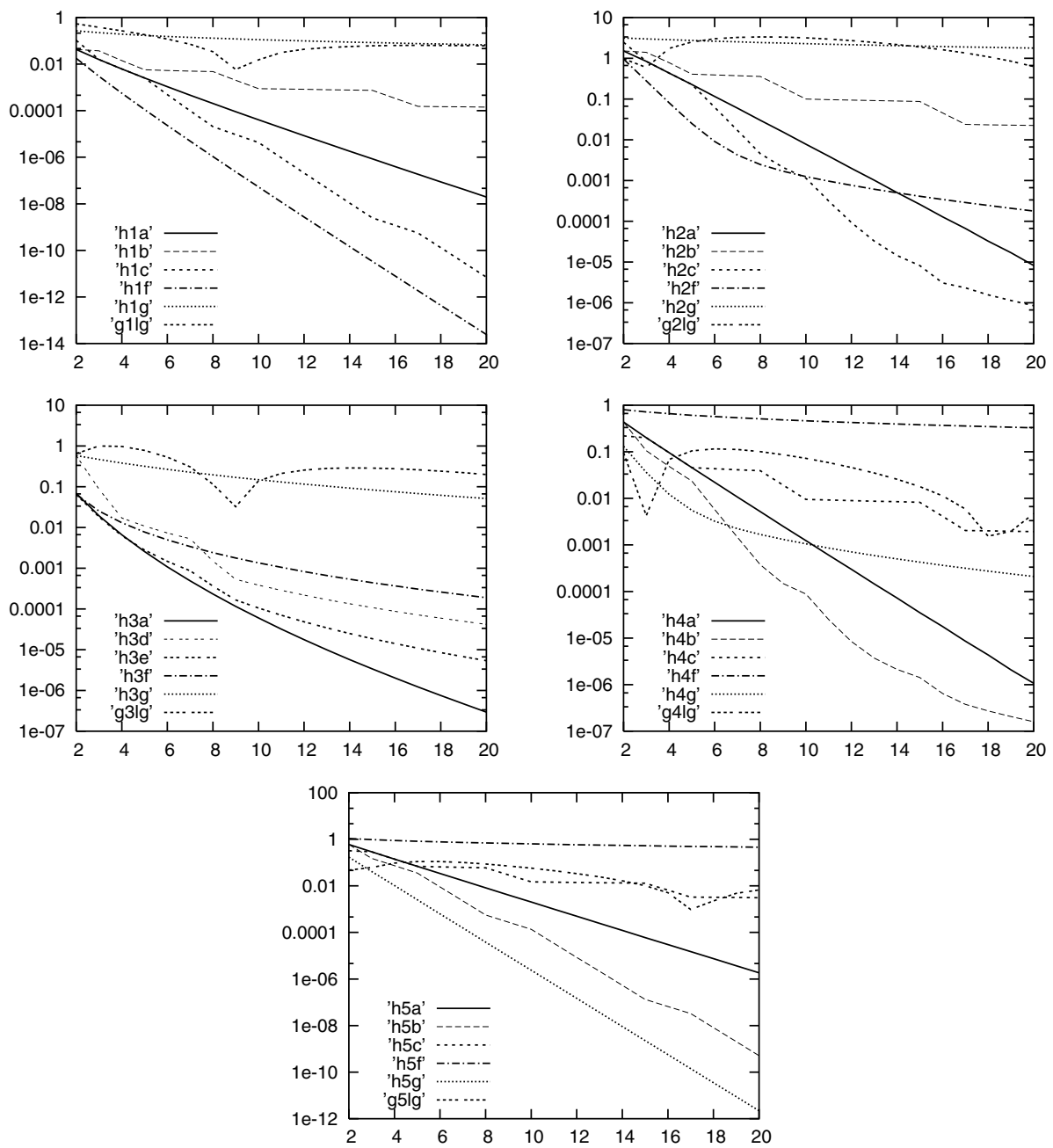

Figure 3. Errors as a function of $n$ for Example 6.3

As a general conclusion we can say that L-orthogonal quadrature formulas give excellent numerical results, especially in the presence of singularities either at the origin or at infinity. Furthermore, the influence of the sequence $\{p(n)\}_{n \geq 0}$ is clearly exposed. However, it should also be said that a much deeper computational work needs to be done in order to create algorithms which allow us to compute these formulas in a more efficient way.

\section{ACKNOWLEDGMENTS}

The authors wish to thank the referees for their valuable comments and suggestions. 


\section{REFERENCES}

[1] D.H. Bailey, Y. Hida, X. S. Li, B. Thompson. ARPREC: An Arbitrary Precision Computation Package. Manuscript, 2002.

[2] G.A. Baker Jr., G.S. Rushbrooke, H.E. Gilbert. High-temperature series expansions for the spin- $\frac{1}{2}$ Heisenberg model by the method of irreducible representations of the symmetric group. Phys. Rev. (2) 135 (1964), A1272-A1277. MR0171545 (30:1776)

[3] A. Bultheel, C. Díaz-Mendoza, P. González-Vera, R. Orive. Quadrature on the half line and two-point Padé approximants to Stieltjes functions. II. Convergence. J. Comput. Appl. Math. 77 (1997) 53-76. MR1440004 (98a:41023)

[4] A. Bultheel, C. Díaz-Mendoza, P. González-Vera, R. Orive. Quadrature on the half line and two-point Padé approximants to Stieltjes functions. Part III, The unbounded case. J. Comput. Appl. Math 87(1997) 95-117. MR1488823 (98j:41033)

[5] A. Bultheel, C. Díaz-Mendoza, P. González-Vera, R. Orive. Estimates of the rate of convergence for certain quadrature formulas on the half-line. Continued fractions: from analytic number theory to constructive approximation (Columbia, MO, 1998), Contemp. Math., 236, Amer. Math. Soc., Providence, RI, 1999, 85-100. MR1665364 (2000g:65017)

[6] A. Bultheel, C. Díaz-Mendoza, P. González-Vera, R. Orive. On the convergence of certain Gauss-Type quadrature formulas for unbounded intervals. Math. Comp., 69 (2000), 721-747. MR1651743 (2000i:65034)

[7] A. Bultheel, C. Díaz-Mendoza, P. González-Vera, R. Orive. Orthogonal Laurent polynomials and quadrature formulas for unbounded intervals: I. Gauss-type formulas. Rocky Mountain J. Math. 33 (2003), 585-608. MR2021367 (2005d:41039)

[8] A. Bultheel, P. González-Vera, E. Hendriksen, O. Njåstad. Orthogonal rational function and quadrature on the half-line. J. Complexity 19 (2003), 212-230. MR1984110 (2004d:41054)

[9] A. Bultheel, P. González-Vera, R. Orive. Quadrature on the half-line and two-point Padé approximants to Stieltjes functions. I. Algebraic aspects. Proceedings of the International Conference on Orthogonality, Moment Problems and Continued Fractions (Delft, 1994). J. Comput. Appl. Math. 65 (1995), no. 1-3, 57-72. MR1379119 (96m:41018)

[10] A. Draux. Polynômes orthogonaux formels. Applications. Lecture Notes in Mathematics, 974. Springer-Verlag, Berlin, 1983. MR0690769 (85h:42027)

[11] T.S. Chihara. An Introduction to Orthogonal Polynomials. Gordon and Breach, New York, 1978. MR0481884(58:1979)

[12] L. Cochram, S.C. Cooper. Orthogonal Laurent polynomials on the real line. In S.C. Cooper and W.J. Thron, eds., Continued Fractions and Orthogonal Functions, Marcel Dekker, New York, 1994, 47-100. MR 1263248 (95b:42024)

[13] R. Cruz-Barroso, P. González-Vera. Orthogonal Laurent polynomials and quadratures on the unit circle and the real half-line. Submitted (2003).

[14] G. Freud. Orthogonal polynomials. Pergamon Press, Oxford, 1971.

[15] W. Gautschi. Numerical analysis. An introduction. Birkhäuser Boston, Inc., Boston, MA, 1997. MR1454125 (98d:65001)

[16] P. González-Vera, O. Njåstad. Convergence of two-point Padé approximants to series of Stieltjes. Extrapolation and rational approximation (Luminy, 1989). J. Comput. Appl. Math. 32 (1990), no. 1-2, 97-105. MR1091780 (92k:41021)

[17] P.E. Gustafson and B.A. Hagler. Gaussian quadrature rules and numerical examples for strong extension of mass distribution functions. J. Comput. Appl. Math 105 (1999), no. 1-2, 317-326. MR.1690598 (2000d:42012)

[18] E. Hendriksen. A characterization of classical orthogonal Laurent polynomials. Nederl. Akad. Wetensch. Indag. Math. 50 (1988), no. 2, 165-180. MR0952513 (89h:33014)

[19] E. Hendriksen, H. van Rossum. Orthogonal Laurent polynomials. Nederl. Akad. Wetensch. Indag. Math. 48 (1986), no. 1, 17-36. MR0834317 (87j:30008)

[20] E. Hille. Analytic function theory. Vol. II. Introductions to Higher Mathematics, Ginn and Co., Boston, Mass.-New York-Toronto, Ont., 1962. MR0201608 (34:1490)

[21] J. Illán, G. López Lagomasino. Sobre los métodos interpolatorios de integración numérica y su conexión con la approximación racional. Rev. Cienc. Mat., 8 (1987), 31-44. MR0939222 (89b:65049)

[22] W.B. Jones, O. Njåstad, W.J. Thron. Two-point Padé expansions for a family of analytic functions. J. Comput. Appl. Math. 9 (1983), no. 2, 105-123. MR0709210 (84j:30057) 
[23] W.B. Jones, O. Njåstad. Orthogonal Laurent polynomials and strong moment theory: a survey. Continued fractions and geometric function theory (CONFUN) (Trondheim, 1997). J. Comput. Appl. Math. 105 (1999), no. 1-2, 51-91. MR1690578 (2000d:30054)

[24] W.B. Jones, W.J. Thron. Two-point Padé tables and T-fractions. Bull. Amer. Math. Soc. 83 (1977), no. 3, 388-390. MR0447543 (56:5853)

[25] W.B. Jones, W.J. Thron. Orthogonal Laurent polynomials and gaussian quadrature. Quantum Mechanics in Mathematics, Chemistry, and Physics (K.E. Gustafson and W.P. Reinhardt, eds.), Plenum Press, New York, 1981, 449-455.

[26] W.B. Jones, W.J. Thron, H. Waadeland. A strong Stieltjes moment problem. Trans. Amer. Math. Soc. 261 (1980), 503-528. MR0580900 (81j:30055)

[27] G. López Lagomasino, A. Martínez Finkelshtein. Rate of convergence of two-point Padé approximants and logarithmic asymptotics of Laurent-type orthogonal polynomials. Constr. Approx. 11 (1995), 255-286. MR1342387 (96i:41016)

[28] A. Magnus. On the structure of the two-point Padé table. Analytic theory of continued fractions (Loen, 1981), Lecture Notes in Math., 932, Springer, Berlin-New York, 1982, 176-193. MR0690461 (84c:30058)

[29] J. H. McCabe. A formal extension of the Padé table to include two point Padé quotients. J. Inst. Math. Appl. 15 (1975), 363-372. MR0381246 (52:2143)

[30] O. Njåstad, W.J. Thron. The theory of sequences of orthogonal L-polynomials. In H. Waadeland \& H. Wallin, eds., Padé Approximants and Continued fractions, Det Kongelige Norkse Videnskabers Selskab Skrifter (No. 1), 1983, 54-91.

[31] A. Sri Ranga. Another quadrature rule of highest algebraic degree of precision. Numer. Math. 68 (1994), 283-294. MR 1283343 (95c:65047)

[32] A. Sri Ranga. Symmetric orthogonal polynomials and the associated orthogonal Lpolynomials. Proc. Amer. Math. Soc. 123 (1995), no. 10, 3135-3141. MR1291791|(95m:42035)

[33] A. Sri Ranga, W. Van Assche. Blumenthal's theorem for Laurent orthogonal polynomials. J. Approx. Theory 117 (2002), no. 2, 255-278. MR1903057 (2003g:42044)

[34] T.J. Stieltjes. Recherches sur les fractions continues. Ann. Fac. Sci. Univ. Toulouse 8 (1984), 1-122; 9 (1985), 1-47. MR1607517 (99d:01040) MR.1623484 (99d:01041)

Department of Mathematical Analysis, La Laguna University, 38271 La Laguna, Tenerife, Canary Islands, Spain

E-mail address: cjdiaz@ull.es

Department of Mathematical Analysis, La Laguna University, 38271 La Laguna, Tenerife, Canary Islands, Spain

Department of Mathematical Analysis, La Laguna University, 38271 La Laguna, Tenerife, Canary Islands, Spain 